\title{
Coevolution of black hole accretion and star formation in galaxies up to $z=3.5$
}

\author{
R. Carraro ${ }^{1,2}$, G. Rodighiero ${ }^{2,3}$, P. Cassata ${ }^{2,3}$, M. Brusa ${ }^{4,5}$, F. Shankar ${ }^{6}$, I. Baronchelli ${ }^{2}$, E. Daddi ${ }^{7}$, I. Delvecchio ${ }^{7}$,
} A. Franceschini ${ }^{2}$, R. Griffiths ${ }^{8,9}$, C. Gruppioni ${ }^{5}$, E. López-Navas ${ }^{1}$, C. Mancini ${ }^{2}$, S. Marchesi ${ }^{10,5}$, M. Negrello ${ }^{11}$, A. Puglisi ${ }^{12}$, E. Sani ${ }^{13}$, and H. Suh ${ }^{14, \star}$

${ }^{1}$ Instituto de Física y Astronomía, Universidad de Valparaíso, Gran Bretaña 1111, Playa Ancha, Valparaíso, Chile e-mail: carrarorosamaria@gmail.com

2 Dipartimento di Fisica e Astronomia, Università di Padova, Vicolo dell'Osservatorio, 3, 35122 Padova, Italy

3 INAF Osservatorio Astronomico di Padova, vicolo dell'Osservatorio 5, 35122 Padova, Italy

${ }^{4}$ Dipartimento di Fisica e Astronomia, Università di Bologna, via Gobetti 93/2, 40129 Bologna, Italy

5 INAF - Osservatorio di Astrofisica e Scienza dello Spazio di Bologna, Via Piero Gobetti, 93/3, 40129 Bologna, Italy

${ }^{6}$ Department of Physics and Astronomy, University of Southampton, Highfield SO17 1BJ, UK

7 CEA, IRFU, DAp, AIM, Université Paris-Saclay, Université Paris Diderot, Sorbonne Paris Cité, CNRS, 91191 Gif-sur-Yvette, France

8 Physics \& Astronomy Dept., University of Hawaii at Hilo., 200 W. Kawili St., Hilo, HI 96720, USA

9 Physics Dept., Carnegie Mellon University, Pittsburgh, PA 15213, USA

10 Department of Physics and Astronomy, Clemson University, Kinard Lab of Physics, Clemson, SC 29634, USA

11 School of Physics and Astronomy, Cardiff University, The Parade, Cardiff CF24 3AA, UK

12 Center for Extragalactic Astronomy, Durham University, South Road, Durham DH1 3LE, UK

13 European Southern Observatory, Alonso de Cordova 3107, Casilla 19, Santiago 19001, Chile

14 Subaru Telescope, National Astronomical Observatory of Japan (NAOJ), 650 North A'ohoku place Hilo, HI 96720, USA

Received 6 September 2019 / Accepted 20 July 2020

\section{ABSTRACT}

\begin{abstract}
Aims. We study the coevolution between the black hole accretion rate (BHAR) and the star formation rate (SFR) in different phases of galaxy life: main-sequence star-forming galaxies, quiescent galaxies, and starburst galaxies at different cosmic epochs.

Methods. We exploited the unique combination of depth and area in the COSMOS field and took advantage of the X-ray data from the Chandra COSMOS-Legacy survey and the extensive multiwavelength ancillary data presented in the COSMOS2015 catalog, including in particular the UVista Ultra-deep observations. These large datasets allowed us to perform an X-ray stacking analysis and combine it with detected sources in a broad redshift interval $(0.1<z<3.5)$ with unprecedented statistics for normal star-forming, quiescent, and starburst galaxies. The X-ray luminosity was used to predict the black holeAR, and a similar stacking analysis on farinfrared Herschel maps was used to measure the corresponding obscured SFR for statistical samples of sources in different redshifts and stellar mass bins.

Results. We focus on the evolution of the average SFR-stellar mass $\left(M_{*}\right)$ relation and compare it with the BHAR- $M_{*}$ relation. This extends previous works that pointed toward the existence of almost linear correlations in both cases. We find that the ratio between BHAR and SFR does not evolve with redshift, although it depends on stellar mass. For the star-forming populations, this dependence on $M_{*}$ has a logarithmic slope of $\sim 0.6$ and for the starburst sample, the slope is $\sim 0.4$. These slopes are both at odds with quiescent sources, where the dependence remains constant $(\log (\mathrm{BHAR} / \mathrm{SFR}) \sim-3.4)$. By studying the specific BHAR and specific SFR, we find signs of downsizing for $M_{*}$ and black hole mass $\left(M_{\mathrm{BH}}\right)$ in galaxies in all evolutionary phases. The increase in black hole massdoubling timescale was particularly fast for quiescents, whose super-massive black holes grew at very early times, while accretion in star-forming and starburst galaxies continued until more recent times.

Conclusions. Our results support the idea that the same physical processes feed and sustain star formation and black hole accretion in star-forming galaxies while the starburst phase plays a lesser role in driving the growth of the supermassive black holes, especially at high redshift. Our integrated estimates of the $M_{*}-M_{\mathrm{BH}}$ relation at all redshifts are consistent with independent determinations of the local $M_{*}-M_{\mathrm{BH}}$ relation for samples of active galactic nuclei. This adds key evidence that the evolution in the BHAR/SFR is weak and its normalization is relatively lower than that of local dynamical $M_{*}-M_{\mathrm{BH}}$ relations.
\end{abstract}

Key words. galaxies: evolution - galaxies: active - galaxies: starburst - galaxies: star formation - X-rays: galaxies

\section{Introduction}

Observational studies and cosmological simulations have revealed a deep interconnection between galaxies and their central supermassive black hole (SMBH): they appear to coevolve and affect each other during their lives. It has been reported

^ Subaru Fellow. several times that the SMBH mass correlates with a number of galaxy properties, including bulge mass, velocity dispersion, and Sérsic index. The relation with the velocity dispersion appears to the most fundamental relation so far (e.g., Bernardi et al. 2007; Shankar et al. 2016, 2017, 2019).

Coincidentally, the cosmic star formation rate density (SFRD) and black hole accretion rate densities (BHARD) share 
a similar evolution: they reach a peak of activity at redshift $z \sim 2$ and then decrease to the present epoch (Boyle \& Terlevich 1998; Delvecchio et al. 2014; Madau \& Dickinson 2014). Additionally, semianalytic models and hydrodynamic simulations show that a self-regulating mechanism is required between the star formation and the black hole accretion in order to reproduce local scaling relations (see Somerville \& Davé 2015, for a review).

The history of star formation in galaxies throughout the life of the Universe has been thoroughly constrained in recent years (see Madau \& Dickinson 2014, for a review): the SFRD of the Universe increased by a factor of about 10 since $z \sim 8$, reached a peak at around $z \sim 2$, and declined by a factor of 10 since then. This decline is thought to depend on the decreasing availability of cold gas that is required to form stars (e.g., Feldmann et al. 2016). In addition, we know that most galaxies follow a sequence on the stellar mass $\left(M_{*}\right)$ - star formation rate (SFR) plane with an almost linear slope. The so-called main-sequence of star-forming galaxies (Brinchmann et al. 2004; Daddi et al. 2007; Elbaz et al. 2007; Noeske et al. 2007; Rodighiero et al. 2014; Aird et al. 2017) suggests that most of the cosmic star formation takes place through secular processes (Rodighiero et al. 2011) because it has been observed at all redshifts and shows no evolution in its slope, but an increasing normalization with redshift (Tasca et al. 2015; Schreiber et al. 2015; Tomczak et al. 2016): at earlier epochs, galaxies of a given stellar mass were forming more stars than in the local Universe. We also find that a number of galaxies lie substantially above and below the MS. Starburst galaxies are a small fraction of star-forming galaxies ( $\sim 2 \%$ Rodighiero et al. 2011), and their SFRs and gas fractions are higher than those on the MS. Quiescent galaxies, on the other hand, lie below the main-sequence and therefore have little star formation. They have been shown to evolve from $z \sim 1.8$, where they have significant amounts of dust and gas $(\sim 5-10 \%)$ but their star formation efficiency is low, to the local universe, where they are gas poor (Gobat et al. 2018).

Just like star formation, black hole accretion depends on the availability of cold gas. Simulations show that gas flows into galaxies, where it cools to eventually fuel star formation and black hole accretion (Hopkins \& Quataert 2010). This fueling by gas is expected to be even more pronounced in starburst galaxies, many of which are likely to undergo a major merging event (Di Matteo et al. 2005; Hopkins et al. 2008): during the early stages of galaxy merging, gas can efficiently cool and lose angular momentum, eventually feeding central star formation and black hole growth. The black hole accretion is initially obscured by thick layers of dust, which are then possibly removed by its increasing radiation and momentum feedback, revealing the quasar. Eventually, the gas is consumed, the quasar luminosity fades rapidly, and the star formation episode ceases. This leaves a "red and dead" elliptical galaxy with no or very little star formation or black hole accretion (Granato et al. 2004; Lapi et al. 2006).

This picture might suggest that just like the majority of galaxies follow a main-sequence in the SFR- $M_{*}$ plane, a similar relation between the black hole accretion rate (BHAR) and the $M_{*}$ might exist: the more massive the galaxy, the higher the availability of inflowing gas for star formation and black hole accretion, which would mean that they both should correlate with stellar mass. In addition, galaxies offset from the mainsequence (starbursts and quiescents), might have a BHAR that varies accordingly with the gas that is typically available in that phase (Rodighiero et al. 2019).

In order to search for these potential correlations, many authors have used the X-ray luminosity of galaxies as a proxy of BHAR: X-rays are very energetic photons that are created very close to the central SMBH, and other contaminants in the host galaxies at these wavelengths, for example, emission from stellar processes or binary systems, are usually less powerful and not dominant (see the review by Brandt \& Alexander 2015). Nevertheless, the first studies that traced the instantaneous BHAR with X-ray flux failed at finding any BHAR- $M_{*}$ relation (Silverman et al. 2009; Shao et al. 2010; Mullaney et al. 2012a; Rosario et al. 2012; Azadi et al. 2015). A lack of a correlation between SFR and BHAR does not by itself necessarily imply a lack of physical connection. It might arise, for example, from different duty cycles and variabilities that characterize the two processes. Episodes of star formation last for several Gyr, while the SMBH duty cycles are believed to be very short, with accretion episodes of about $10^{5} \mathrm{yr}$ and variability timescales that range from minutes to months.

In order to constrain more robust and reliable BHAR, it is thus necessary to average their growth rate over a long time interval. A very promising technique to achieve this goal consists of stacking X-ray images. Stacking allows us to perform studies on mass-complete samples by averaging the count rates of the $\mathrm{X}$-ray images in the optical positions of the galaxies, thus increasing the signal-to-noise ratio, and allowing us to reach fluxes well below the single-source detection threshold of the observations. Moreover, because the BHAR is a stochastic event, stacking large samples of galaxies in a given volume is equivalent to averaging the growth rate of all galaxies. Previous works indeed searched for a relation between $M_{*}$ and average BHAR by stacking X-ray images (e.g., Mullaney et al. 2012b; Rodighiero et al. 2015; Yang et al. 2017), and the probability distribution of specific X-ray luminosity with a maximum likelihood approach (Aird et al. 2012; Bongiorno et al. 2012; Yang et al. 2018) and a Bayesian approach (Aird et al. 2018). All studies point toward a positive correlation between the BHAR and the $M_{*}$ for star-forming galaxies, very similar to the mainsequence of star-forming galaxies, with a slope close to unity, non-negligible redshift evolution, a positive slope for the BHARto-SFR ratio as a function of stellar mass, and indications of different behaviors for quiescent and starburst galaxies. Thus far, no study has presented a complete analysis throughout all galaxy life phases by highlighting the evolution of the accretions and their ratio throughout cosmic time. This is what we present here. So far, only Aird et al. (2019) have shown that the fraction of active galactic nucleus (AGN) galaxies is higher below the mainsequence and in starbursts.

We here characterize the evolution of the average BHAR for normal star-forming, quiescent, and starburst galaxies at $0.1<z<3.5$. This redshift interval encompasses the majority of the history of the Universe and contains two crucial epochs in its evolution: the peak of the star formation rate density and BHAR of the Universe at $z \sim s 2$, and their decline to the local Universe. We take advantage of the unique depth, area, and wavelength coverage of the COSMOS field, which allows us to select a mass-complete sample with large statistics out to very high redshifts for each galaxy phase. This is particularly important for starbursts, which are rare objects and require a large field in order to be found in good numbers for statistics. We apply the stacking technique to X-ray images from the Chandra COSMOS-Legacy survey (Civano et al. 2016) and combine the results with the actual X-ray detections in order to estimate the average X-ray luminosity and therefore average BHAR. We compare the evolution of the average BHAR with that of the average SFR. We show that these data confirm and extend previous claims about the evolution of the specific 
accretions and that the ratio of BHAR to SFR and $M_{*}$ are correlated. More specifically, in Sect. 2 we introduce the parent data sample we used for this work, from which we select the sample as described in Sect. 3. In Sect. 4 we describe the method we used to estimate the average X-ray luminosities, BHAR and SFR. In Sects. 5 and 6 we describe our results and discuss them. In Sect. 7 we describe the derivation of specific BHAR and specific SFR and discuss the respective results. In Sect. 8 we compare our $M_{\mathrm{BH}}-M_{*}$ relation to observational and simulation relations. Finally, in Sect. 9 we enumerate the conclusions of this work.

Throughout this paper we use a Chabrier (2003) initial mass function (IMF) assuming a flat cosmology with $H_{0}=70, \Omega_{\lambda}=$ $0.7, \Omega_{0}=0.3$.

\section{Data}

Our study focuses on the relation between average BHAR, SFR, and $M_{*}$ across a wide redshift range, from $z=0.1$ to $z=3.5$, and across different evolutionary stages of galaxies: (i) normal starforming galaxies, which accrete gas secularly to slowly form new stars, and in which the bulk of the cosmic star formation took place; (ii) starburst galaxies, which are a small fraction of all galaxies at all epochs ( $2 \%$ Rodighiero et al. 2011; but see also Caputi et al. 2017) and are going through a great burst of star formation that drives them significantly above the main-sequence galaxies on the SFR- $M_{*}$ plane; and (iii) quiescent galaxies, which form stars at a very slow pace.

Star-forming and quiescent galaxies can be easily selected at all redshifts from their emission in the optical/near-infrared (NIR) rest-frame bands, whereas starburst galaxies are heavily obscured in the optical and are therefore more easily identified by the thermal emission from the dust in the far-infrared (FIR). Therefore we selected our samples of star-forming and quiescent galaxies from the catalog by Laigle et al. (2016), which is NIR selected, while we used the FIR-selected catalog by Gruppioni et al. (2013) to identify starburst galaxies. For the BHAR we used the catalog by Civano et al. (2016), obtained from the Chandra COSMOS-Legacy program, and complemented it by stacking on their X-ray images. We estimated the SFR for star-forming and quiescent galaxies by combining FIR stacking on Herschel images and detections with UV luminosity. We present in this section the catalogs we used for the sample selection and the data we used to estimate the average BHAR and SFR.

\subsection{Optical-NIR catalog}

We selected our sample of star-forming and quiescent galaxies in the COSMOS field (Scoville et al. 2007) from the COSMOS 2015 catalog (Laigle et al. 2016), which uses photometry from UV (Galex) to the mid-infrared (MIR, IRAC). This catalog constitutes the UltraVISTA DR2.

The multiwavelength catalog includes UV photometry in the far-UV and near-UV (NUV) bands with the GALEX satellite (Zamojski et al. 2007); UV/optical photometry in the $\mathrm{u}^{*}$-band from the Canada-France-Hawaii Telescope (CFHT/MegaCam); optical photometry from the COSMOS-20 survey, which is composed of 6 broad bands $(B, V, g, r, i, z+), 12$ medium bands (IA427, IA464, IA484, IA505, IA527, IA574, IA624, IA679, IA709, IA738, IA767, and IA827), and 2 narrow bands (NB711 and NB816), taken with the Subaru Suprime- Cam (Taniguchi et al. 2007, 2015); new and deeper $z^{++}$and $Y$-band data, both taken with the Hyper-Suprime-Cam (HSC) on Subaru; $\mathrm{H}$ and $K_{s}$ NIR photometry obtained with WIRcam/CFHT
(McCracken et al. 2010), as well as deeper $J, H$, and $K_{S}$ imaging obtained with VIRCAM/VISTA UltraVISTA (McCracken et al. 2012) in the central $1.5 \mathrm{deg}^{2}$ of the COSMOS field (the coverage by UltraVISTA is not homogeneous, with alternating "deep" and "ultradeep" stripes that reach depths of 24 and 24.7 in $K_{S}$-band, respectively); NIR data from IRAC, as part of the SPLASH COSMOS, together with S-COSMOS (Sanders et al. 2007); MIR and FIR data with Spitzer IRAC and MIPS (Multiband Imaging Photometer), from the Spitzer Extended Mission Deep Survey and the Spitzer-Candels survey (Ashby et al. 2015) data, among others; FIR from the Herschel PACS and SPIRE instruments, taken as part of the PACS Evolutionary Probe (PEP) guaranteed-time key program (Lutz et al. 2011), the largest field of the program, observed for about $200 \mathrm{~h}$ to a $3 \sigma$ depth at $160 \mu \mathrm{m}$ of $10.2 \mathrm{mJy}$ and at $100 \mu \mathrm{m} \sim 5 \mathrm{mJy}$.

The point spread function (PSF) of the VISTA bands was estimated in each photometric band by modeling isolated known stars from the COSMOS ACS/HST catalog (Koekemoer et al. 2007; Leauthaud et al. 2007) with the PSFEX tool (Bertin 2013); The target PSF was chosen in order to minimize the applied convolutions, and it is the desired PSF of all bands after homogenization. The required convolution kernel was calculated in each band by finding the kernel that minimizes the difference between the target PSF and the convolution product of this kernel with the current PSF. The images were then convolved with this kernel.

Then, a $\chi^{2}$ detection image (Szalay et al. 1999), produced by combining NIR images of UltraVISTA $\left(Y J H K_{S}\right)$ with the optical $z^{++}$-band data from Subaru, was used to identify objects; the photometry for these objects in the other bands was obtained by running SEXTRACTOR in dual-image mode. Fluxes were extracted from $2^{\prime \prime}$ to $3^{\prime \prime}$ diameter apertures on PSF-homogenized images in each band, except for a few cases: for GALEX, the fluxes were measured using a PSF fitting method with the $u^{*}$ band image used as a prior, and for the SPLASH IRAC imaging, the IRACLEAN tool (Hsieh et al. 2012) was used to derive the photometry using the UltraVISTA $z Y J H K_{s} \chi^{2}$ image as a prior. Photometry at $24 \mu \mathrm{m}$ was obtained from the COSMOS MIPSselected band-merged catalog (Le Floc'h et al. 2009). Far-IR photometry is provided by Herschel PACS (PEP guaranteedtime program, Lutz et al. 2011) and SPIRE (HERMES consortium, Oliver et al. 2012) at 100, 160, 250, 350, and $500 \mu \mathrm{m}$.

The Laigle et al. (2016) catalog provides secondary products as well, including photometric redshifts, stellar masses, and star formation rates. In particular, photometric redshifts were computed with Le Phare (Arnouts et al. 2002; Ilbert et al. 2006) with the same method as was used in Ilbert et al. (2013): the spectral energy distributions (SED) in 3 arcsec apertures were fit to a set of 31 templates, including spiral and elliptical galaxies from Polletta et al. (2007) and a set of 12 templates of young blue star-forming galaxies, produced using the models of Bruzual \& Charlot (2003). Photometric redshifts for objects that are detected in X-rays were instead taken from Marchesi et al. (2016), who applied a set of templates that is more suitable for X-ray detected galaxies, in which the central black hole might significantly affect the UV/optical photometry (see sect. 2.3). Photometric redshift precision was characterized by comparing it with spectroscopic samples from the COSMOS spectroscopic master catalog (Salvato et al., in prep.).

Stellar masses were derived using Le Phare, with the same method as presented in Ilbert et al. (2015): a Chabrier (2003) initial mass function was assumed, and the photometry was fit with a library of synthetic spectra generated using the stellar population synthesis model of Bruzual \& Charlot (2003), assuming both exponentially declining star formation histories (SFH) 
and delayed SFH $\left(\tau^{-2} t e^{-t / \tau}\right)$, assuming two different metallicities (solar and half-solar). Emission lines were added following the prescription in Ilbert et al. (2009) together with two attenuation curves (the starburst curve of Calzetti et al. 2000 and a curve with a slope $\lambda^{0.9}$ from Appendix A of Arnouts et al. 2013). The $E(B-V)$ values were allowed to vary in a range between 0 and 0.7. The masses were then obtained as the median of the marginalized probability distribution function (PDF).

\subsection{IR data}

We selected the starburst galaxies sample for this work from a FIR-selected catalog. In particular, we used the Gruppioni et al. (2013) catalog, which is selected from PACS/Herschel PEP observations in the COSMOS field. This catalog was also matched with the deep $24 \mu \mathrm{m}$ imaging of Le Floc'h et al. (2009), with the HerMES extragalactic survey (Oliver et al. 2012) observed with SPIRE at 250, 350, and $500 \mu \mathrm{m}$ in the same fields that were covered by PEP and with the IRAC-based catalog of Ilbert et al. (2010), including optical and NIR photometry and photometric redshifts.

Gruppioni et al. (2013) used as reference the blind catalogs at 100 and $160 \mu \mathrm{m}$ from Berta et al. (2010, 2011), selected down to the $3 \sigma$ level, which in COSMOS contain 5355 and 5105 sources at 100 and $160 \mu \mathrm{m}$, respectively. Then they associated their sources with the ancillary catalogs by means of a multiband likelihood ratio technique (Sutherland \& Saunders 1992; Ciliegi et al. 2001), starting from the longest available wavelength (160 $\mu \mathrm{m}$, PACS $)$ and progressively matching $100 \mu \mathrm{m}$ (PACS) and $24 \mu \mathrm{m}$ (MIPS). Their final catalog consists of 4110 and 4118 sources at 100 and $160 \mu \mathrm{m}$, respectively. Spectroscopic or photometric redshifts are available for 3817 and 3849 of their sources, respectively, which they used for the SED fitting in order to estimate the FIR luminosity function.

Stellar masses were obtained by fitting the broadband SED with a modified version of MAGPHYS (da Cunha et al. 2008), which simultaneously fits the broadband UV-to-FIR observed SED of each object and ensures an energy balance between the absorbed UV light and the light that is reemitted in the FIR regime. The redshift for each object was fixed to the spectroscopic redshift when available or else to the photometric redshift; then the SED was fit to a best-fit model that we selected from a library built by combining different SFH, metallicities, and dust contents. Each SFH is the combination of an exponentially declining SFR model, to which random bursts of star formation are superimposed (see da Cunha et al. 2008, 2010). The emission of a possible AGN component was taken into account using a modified version of the MAGPHYS code (SED3FIT, Berta et al. 2013), which adds a torus component to the modeled SED emission by combining the da Cunha et al. (2008) original code with the Fritz et al. (2006) AGN torus library (see also Feltre et al. 2012).

In order to estimate the SFR of their sample, Gruppioni et al. (2013) calculated an SED using all the available multiwavelength data by performing a $\chi^{2}$ fit using the Le Phare code (Arnouts et al. 2002; Ilbert et al. 2006) with the semiempirical template library of Polletta et al. (2007), which is representative of different classes of IR galaxies and AGN. They also added some modified templates in the FIR to better reproduce the observed Herschel data (see Gruppioni et al. 2010), and three starburst templates from Rieke et al. (2009). Then they integrated the best-fitting SED of each source over $8 \leq$ $\lambda_{\text {rest }} \leq 1000 \mu \mathrm{m}$ to derive the total IR luminosities $\left(L_{\mathrm{IR}}=\right.$ $L[8-1000 \mu \mathrm{m}])$ in 11 redshift bins $(0.0-0.3,0.3-0.45,0.45-.6$,
$0.6-0.8,0.8-1.0,1.0-1.2,1.2-1.7,1.7-2.0,2.0-2.5,2.5-3.0$, and 3.0-4.2). Finally, they estimated the $S_{F R}$ for all these sources from the total IR luminosity after subtracting the AGN contribution and using the Kennicutt (1998) relation (see also Gruppioni et al. 2015, for more details).

\subsection{X-ray data}

We used X-ray data from the Chandra COSMOS Legacy Survey (COSMOS-Legacy, Civano et al. 2016), a 4.6 Ms Chandra program that combines new observations obtained during Chandra Cycle 14 with the previous C-COSMOS Survey, allowing the X-ray data to uniformly cover the whole $2.2 \mathrm{deg}^{2}$ of the COSMOS field. The limiting fluxes are $2.2 \times 10^{-16}, 1.5 \times 10^{-15}$, and $8.9 \times 10^{-16} \mathrm{erg} \mathrm{cm}^{-2} \mathrm{~s}^{-1}$ in the $0.5-2,2-10$, and $0.5-10 \mathrm{keV}$ bands, respectively.

The optical counterparts to the X-ray COSMOS-Legacy sources are available in Marchesi et al. (2016). This was obtained by using the maximum likelihood ratio technique (e.g., Sutherland \& Saunders 1992; Brusa et al. 2005; Civano et al. 2012) by matching X-ray sources to three separate bands: i-band data from Ilbert et al. (2009), $K_{s}$-band data using UltraVISTA DR2, and IRAC 3.6 micron using either SPLASH or Sanders et al. (2007) sources from the Laigle et al. (2016) catalog in the UltraVISTA field. Optical counterparts were crosscorrelated with the master spectroscopic catalog (Salvato et al., in prep.), which contains spectroscopic redshifts from numerous observing campaigns and instruments. For the sources for which no spectroscopic redshift is available, a photometric redshift was provided that we obtained by following the same procedure as in Salvato et al. (2011).

\section{Sample selection}

We selected a mass-complete sample from Laigle et al. (2016) by limiting our selection to the area that is covered by the UltraVISTA-DR2 observations $\left(1.5 \mathrm{deg}^{2}\right)$ in the redshift interval $0.1<z<3.5$. In order to separate star-forming from quiescent galaxies, we used the classification from Laigle et al. (2016), which is based on the rest-frame NUV $-r / r-J$ color-color diagram as in Ilbert et al. (2013). It allows separating dust-obscured galaxies from older stellar populations. In this diagram, galaxies with colors NUV $-r>3(r-J)+1$ and NUV $-r>3.1$ are classified as quiescent.

We selected starburst galaxies from the Gruppioni et al. (2013) catalog in the same redshift interval down to $M_{*} \sim$ $10^{9.5} M_{\odot}$. Again, we chose this catalog because it is FIR selected, that is, selected based on the specific star formation rate (at least to a first approximation), and includes $\mathrm{SFR}_{\mathrm{IR}}$ estimates, which allowed us to robustly estimate the SFR of highly starforming galaxies that are heavily obscured. Based on Fig. 15 of Gruppioni et al. (2013), above $z \sim 1.3$ we probably miss the lowSFR starburst sources, therefore we decided to show these data points as upper limits. The starburst galaxies were selected to have an SFR that is at least four times higher than the SFR of a typical main-sequence galaxy with a similar stellar mass and redshift, which is our definition of starburst galaxy. For the purpose of their selection, we considered the parameterization of the main-sequence from Schreiber et al. (2015) and obtained the relation

$$
\begin{aligned}
\operatorname{SFR}_{\mathrm{IR}}^{\mathrm{SB}} & \geq 4 \times \operatorname{SFR}_{\mathrm{MS}}\left(z_{\mathrm{ave}}, M_{*}\right) \\
& =4 \times\left[m-m_{0}+a_{0} r-a_{1}\left[\max \left(0, m-m_{1}-a_{2} r\right)\right]^{2}\right],
\end{aligned}
$$


R. Carraro et al.: Coevolution of black hole accretion and star formation in galaxies up to $z=3.5$

Table 1. Number of X-ray (2-7 keV band) stacked and detected galaxies per redshift bin and galaxy type.

\begin{tabular}{|c|c|c|c|c|c|c|}
\hline \multirow[b]{2}{*}{ Redshift bins } & \multicolumn{2}{|c|}{ Star-forming } & \multicolumn{2}{|c|}{ Quiescent } & \multicolumn{2}{|c|}{ Starburst } \\
\hline & Detections & Stacked & Detections & Stacked & Detections & Stacked \\
\hline $0.10<z<0.65$ & 249 & 8211 & 45 & 1968 & 12 & 66 \\
\hline $0.65<z<1.30$ & 743 & 28037 & 116 & 7055 & 22 & 366 \\
\hline $1.30<z<2.25$ & 764 & 31012 & 42 & 2835 & 48 & 280 \\
\hline $2.25<z<3.50$ & 337 & 11615 & 16 & 443 & 9 & 115 \\
\hline
\end{tabular}

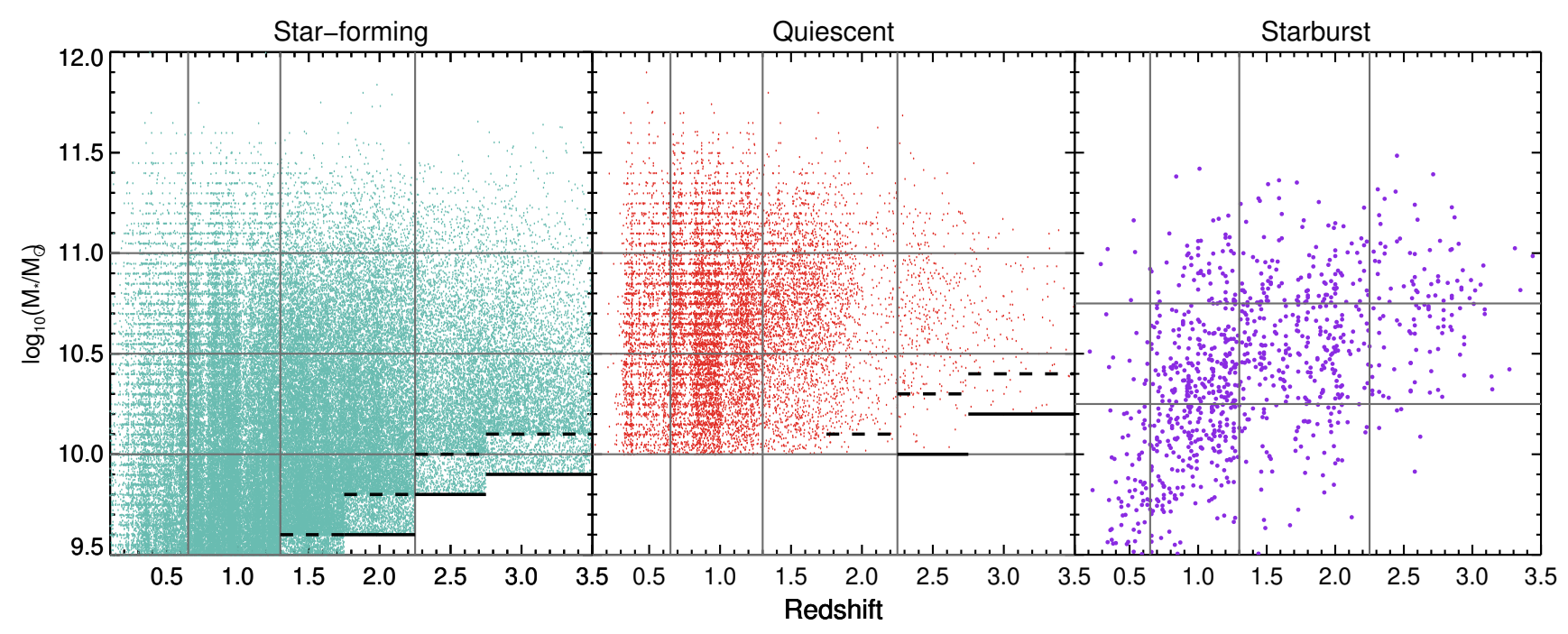

Fig. 1. Stellar mass of our sample of galaxies shown as a function of redshift. Star-forming and quiescent galaxies are selected from Laigle et al. (2016) (green and red data points in the left and central panel, respectively), while the starbursts are selected from Gruppioni et al. (2013) (violet data points in the right panel). The solid and dashed black lines are the mass completeness thresholds are for UVista deep and ultradeep stripes from Laigle et al. (2016) for star-forming and quiescent galaxies. For starburst galaxies we considered as mass incomplete the low-mass bins $\left(9.50<\log _{10}\left(M_{\odot} / M_{*}\right)<10.25\right)$ at $z>1.30$. The solid gray horizontal and vertical lines show the limits of our mass and redshift bins.

where $\mathrm{SFR}_{\mathrm{IR}}^{\mathrm{SB}}$ is the star formation rate of a starburst galaxy obtained from its IR luminosity, $r=\log _{10}(1+z), m=$ $\log _{10}\left(M_{*} / 10^{9} M.\right)$, and the parameters $m_{0}=0.5, m_{1}=0.36$ $a_{0}=1.5$, and $a_{1}=0.3$. We used $\mathrm{SFR}_{\mathrm{IR}}^{\mathrm{SB}}, M_{*}$, and $z$ from the Gruppioni et al. (2013) catalog. We matched the starburst sample with the catalog of star-forming and passive galaxies that was previously color-color selected from Laigle et al. (2016), using a 3.5" association radius in order to exclude the starburst galaxies from our star-forming and quiescent selection. For a distance greater than $2^{\prime \prime}$ we only accepted matches that have a $\Delta z=$ $\left|z_{\text {Gruppioni }}-z_{\text {Laigle }}\right|<0.3$, allowing us to include seven more starburst galaxies in our sample. We compared the stellar masses of the starburst sample (derived from MAGPHYS) with their stellar masses from Laigle et al. (2016) (derived from Le Phare) in each of the redshift bins and found that they lie along the 1:1 relation with increasing scatter with redshift (RMS ranging from $0.18 \mathrm{dex}$ at low $z$ to 0.35 dex at high- $z$ ), but with no offset (the median of the difference of the masses is about $10^{-2}$ ). From the cross-match we find that 965 color-selected star-forming galaxies are IR-classified as starbursts (this is expected because the NUV $-r / r-J$ diagram does not have a starburst area) and 28 color-selected quiescents are IR starbursts. We moved 1494 galaxies that are $24 \mu \mathrm{m}$ MIPS/Spitzer detected from our quiescent selection to the starforming galaxy sample because the IR emission indicates that a certain amount of optically hidden star formation is ongoing. For the X-ray detected star-forming and quiescent galaxies we used the redshift included in Marchesi et al. (2016). We have a final number of 83904 star-forming, 12839 quiescent, and 1003 starburst galaxies.
We further divided the sample into four redshift bins: $0.1<$ $z<0.65,0.65<z<1.3,1.3<z<2.25$, and $2.25<z<3.5$.

Every redshift bin was chosen with the purpose of including a sufficient number of galaxies (see Table 1), and then the galaxy sample of each redshift bin was divided into mass bins. Our final sample is shown in Fig. 1 together with the mass-completeness thresholds from Laigle et al. (2016) for star-forming and quiescent galaxies and the limits of our stellar mass and redshift bins.

\section{Method}

The aim of this paper is to study the average BHAR for galaxies with different star formation activities, from passive galaxies to starbursts, at different cosmic epochs and at different stellar masses. Because AGN activity is a stochastic event, as the AGN duty cycle is short compared to the star formation episodes in galaxies, it is unlikely that a galaxy has been observed at the peak of its black hole accretion. This means that if we limited the analysis to bright X-ray galaxies, we would obtain a biased view of the average AGN activity in galaxies. For this reason, we complement X-ray individual detections with the stacking analysis on Chandra X-ray images for nondetected galaxies.

\subsection{X-ray stacking analysis}

In order to estimate the average X-ray luminosity for each mass and redshift subsample, we followed the same method as in Rodighiero et al. (2015). We used individual X-ray detections from the COSMOS-Legacy catalog (Civano et al. 2016) 
when possible, taking advantage of the match between optical and X-ray sources performed by Marchesi et al. (2016) (see Sect. 2.3). For the non-X-ray detected sources we performed stacking on the same images using the CSTACK tool $\mathrm{v} 4.32^{1}$ (Miyaji \& Griffiths 2008).

We propagated the probability distributions of count rates with Monte Carlo simulations. We focused on the $2-7 \mathrm{keV}$ band and took advantage of the CSTACK bootstrap output. We simulated it $10^{6}$ times by interpolating the inverted cumulative distribution function of the bootstrap probability distribution. For X-ray detections, we instead assumed a Gaussian probability distribution and a $\sigma$ equal to the error on the count rate from the catalog when available. Alternatively, we set the error on the detection to $\sigma=0.25 \times C R(2-7 \mathrm{keV})$. Count-rate distributions from the stacking and detections were converted into standard flux in the $2-10 \mathrm{keV}$ band through the WebPIMMS ${ }^{2}$ conversion factor obtained for Chandra cycle 14 by assuming a power-law spectrum for the AGN with photon index $\Gamma=1.8$ and galactic hydrogen absorption $\left(N_{\mathrm{H}}=2.6 \times 10^{20} \mathrm{~cm}^{-2}\right.$, Kalberla et al. 2005). This value of the photon index $\Gamma$ is compatible with the cosmic X-ray background according to Ananna et al. (2019) for cutoff energies $E_{c}<100 \mathrm{keV}$. We converted fluxes into restframe with a $K$-correction with the shape of $K_{\text {corr }}=(1+z)^{\Gamma-2}$, where $z$ is the average redshift of each subsample and $\Gamma$ is the photon index just introduced.

Because we search for a global average X-ray measurement by combining detections and non-detections in each redshift and mass bin, we applied the following equation to all the fluxes in the probability distribution of each detection and the stacking:

$F_{\text {ave }}=\frac{\sum_{j=1}^{n_{\text {detected }}} F_{j, \text { detected }}+n_{\text {stacked }} \cdot F_{\text {stacked }}}{n_{\text {detected }}+n_{\text {stacked }}}$,

where in each redshift and mass bin, $n_{\text {detected }}$ is the number of detections, $F_{j \text {,detected }}$ is the flux of each detection, $n_{\text {stacked }}$ is the number of undetected objects that were therefore stacked, and $F_{\text {stacked }}$ is the total flux from the stacked objects. This returns a probability distribution of rest-frame flux for each redshift and mass bin. We show in Table 1 the number of stacked and detected galaxies.

\subsection{X-ray luminosity and black hole accretion rate estimate}

We converted the distribution of average rest-frame fluxes $F_{\text {ave }}$ (Eq. (2)) to average luminosities $L_{\text {ave }}$ using the corresponding luminosity distance at the average redshift of the sources included in the bin. We estimated and subtracted the contribution from the stars (young and old stellar populations) in the $2-10 \mathrm{keV}$ range as in Lehmer et al. (2016) using their best-fit values,

$L_{2-10 \mathrm{keV}}\left(M_{*}, z, \mathrm{SFR}\right)\left[\mathrm{erg} \mathrm{s}^{-1}\right]=\alpha_{0}(1+z)^{\gamma} M_{*}+\beta_{0}(1+z)^{\delta} \mathrm{SFR}$,

with $\log \alpha_{0}=29.37, \log \beta_{0}=39.28, \gamma=2.03$, and $\delta=1.31$. This correction has two terms that take into account the X-ray emission from the young stellar populations, that is, high-mass $\mathrm{X}$-ray binaries, whose emission is proportional to the SFR of the galaxy (see Sect. 4.3), and from the old stellar populations, that is, low-mass X-ray binaries, whose emission is proportional to the stellar mass of the galaxy. This is particularly important in

\footnotetext{
1 http://cstack.ucsd.edu/cstack or http://lambic. astrosen . unam.mx/cstack/ developed by Takamitsu Miyaji.

2 http://cxc.harvard.edu/toolkit/pimms.jsp
}

quiescent galaxies. We confirmed the effect of this correction by comparing our results with the corrections from Fornasini et al. (2018) and Aird et al. (2017). The correction from Aird et al. (2017) gives no apparent difference to our results, while the correction from Fornasini et al., which is to be considered an upper limit because it includes some residual AGN emission, has some effect at the lowest redshift that causes some data points to become compatible with zero and a lower normalization by $\sim 0.2$ dex in star-forming and quiescent galaxies. The overall results are not affected by it, however.

In order to correct for the average internal absorption from the galaxy $\left(N_{\mathrm{H}}\right)$, we estimated the hardness ratios (HR) of our sample. We define the HR as

$\mathrm{HR}=\frac{H-S}{S+H}$

where $H$ and $S$ are the median of the distributions of count rates in the $2-7 \mathrm{keV}$ and $0.5-2 \mathrm{keV}$ band, respectively. We compared the estimated HRs of our data with those obtained from models in PyXspec. The model we used is PHABS*(CABS*ZPHABS*PO+APEC), which is composed of a redshifted and absorbed power-law emission with slope $\Gamma=$ 1.8 and hot gas emission at $k T=1 \mathrm{keV}$. The model also includes galactic absorption. The power-law spectrum was normalized because the fluxes we measured and the APEC warm gas component were normalized to equal the emission expected from the SFR of the galaxies (Mineo et al. 2012, Eq. (1)). Finally, we convolved the model with an auxiliary response file (ARF) from Chandra ACIS-I cycle 14 in order to obtain the $H$ and $S$ count rates. All models give two $N_{\mathrm{H}}$ values at which the HRs are compatible with our data. The only exception are star-forming galaxies in the lowest redshift bin, where the modeled spectra are too soft, probably because the $1 \mathrm{keV}$ gas emission is overestimated. In the remaining cases, we find a possible solution at $N_{\mathrm{H}}=10^{22.0-23.2} \mathrm{~cm}^{-2}$ and another at higher $N_{\mathrm{H}}$ values $N_{\mathrm{H}}=10^{23.5-24.8} \mathrm{~cm}^{-2}$, where all the power-law emission has been absorbed and only the hot gas is visible. The two $N_{\mathrm{H}}$ solutions increase with redshift. We decided to use the solution with a lower $N_{\mathrm{H}}$ because we cannot observe galaxies with Comptonthick levels of obscuration. Even though we performed stacking, the number of photons from heavily obscured AGN in the energy range of Chandra is very low and does not dominate our population. Furthermore, our NIR selection may already exclude dust-obscured galaxies, which are thought to be heavily absorbed in the X-rays (Fiore et al. 2008; Corral et al. 2016; Riguccini et al. 2019). A last consideration is that even though many works reported an intrinsic fraction of highly obscured AGN or Compton-thick AGN of about $\sim 30-50 \%$ at various redshifts (Ueda et al. 2014; Vito et al. 2014; Ricci et al. 2015; Ananna et al. 2019), it still is not possible to detect these galaxies in the Chandra X-ray energies. The HRs of our sample do not show a trend with mass, therefore we decided to use the same obscuration correction for all mass bins of a given galaxy type and redshift. We estimated the correction factors using WebPIMMS, and it extends from absorbed to unabsorbed flux in the $2-10 \mathrm{keV}$ band at the average redshift of the bin and with the $N_{\mathrm{H}}$ found from the HR analysis for each galaxy type. These corrections are approximately 5-10\%.

Finally, we considered the median value of the X-ray luminosity distribution as the representative luminosity of each bin and the 5th and 95th percentiles as the lower and upper limits to the uncertainty associated with the X-ray luminosity. We considered values compatible with zero when the 5 th percentile 
assumed a negative value, and in these cases, we show them as upper limits.

We transformed the X-ray binaries and obscurationcorrected X-ray luminosity into a BHAR as in Merloni \& Heinz (2008), Mullaney et al. (2012b), Delvecchio et al. (2014), Rodighiero et al. (2015), and Baronchelli et al. (2018) with the relation

$\operatorname{BHAR}\left(M_{*}, z\right)=\frac{(1-\epsilon) \times L_{\mathrm{bol}}\left(M_{*}, z\right)}{\epsilon c^{2}}$,

where $L_{\text {bol }}$ is the AGN bolometric luminosity obtained using a luminosity-dependent bolometric correction consisting of (i) the bolometric correction used in Yang et al. (2018), which is a modified version of the bolometric correction from Lusso et al. (2012), down to $L_{X}=10^{42.4} \mathrm{erg} \mathrm{s}^{-1}$, and (ii) $k_{\text {bol }}=16$ for lower luminosities as in She et al. (2017). $c$ is the speed of light in vacuum, and $\epsilon$ is the efficiency by which mass is converted into radiated energy in the accretion process. Here we assumed $\epsilon=0.1$ (e.g., Marconi et al. 2004; Mullaney et al. 2012b; Rodighiero et al. 2015; Baronchelli et al. 2018), or that roughly $10 \%$ of the accreted rest-mass is converted into radiant energy, regardless of $M_{\mathrm{BH}}$.

\subsection{Star formation rates: FIR stacking and UV SED fitting}

For star-forming and quiescent galaxies, we estimated the SFR as the sum of the IR and far-UV-observed contributions $\left(\mathrm{SFR}_{\mathrm{IR}+\mathrm{UV}}\right)$, while for starburst galaxies, we only considered the $\mathrm{SFR}_{\mathrm{IR}}$. The $\mathrm{SFR}_{\mathrm{IR}}$ was derived through the empirical calibration of Kennicutt (1998, Eq. (4)) from the total IR luminosity $\left(L_{\mathrm{IR}}\right)$. The $\mathrm{SFR}_{\mathrm{UV}}$ (not corrected for extinction) was inferred, following the prescription of Kennicutt (1998, Eq. (1)), from the rest-frame luminosity at $1600 \AA\left(L_{1600 \AA}\right)$. The two SFRs were then combined as discussed by Nordon et al. (2013), and they were finally converted into the Chabrier (2003) IMF as in Cimatti et al. (2008) by dividing by a factor of 1.7 ,

$$
\begin{aligned}
\operatorname{SFR}_{\mathrm{IR}+\mathrm{UV}}\left[M_{\odot} \mathrm{yr}^{-1}\right] & =\mathrm{SFR}_{\mathrm{UV}}+\mathrm{SFR}_{\mathrm{IR}} \\
& =\left(2.86 L_{1600 \AA}+1.7 L_{\mathrm{IR}}\right) \times \frac{10^{-10}}{1.7}\left[L_{\odot}\right] .
\end{aligned}
$$

In order to obtain the $\mathrm{SFR}_{\mathrm{IR}+\mathrm{UV}}$ for star-forming and quiescent galaxies, we performed bootstrapping, in which we selected a subsample of galaxies in a $M_{*}$ and $z$ bin at each iteration and estimated their $L_{\mathrm{IR}}$ and $L_{1600 \AA}$. We then combined the median of each luminosity through Eq. (6) and thus derived the $\mathrm{SFR}_{\mathrm{IR}+\mathrm{UV}}$ distribution. $L_{\mathrm{IR}}$ was obtained by stacking $160 \mu \mathrm{m}$ PACS/Herschel maps as in Rodighiero et al. (2014). In our procedure we accounted for detections and nondetections to obtain the final median stacked fluxes that we then converted into bolometric luminosities by adopting an average $k$-correction (Chary \& Elbaz 2001). Because the $L_{1600 \AA}$ luminosity is not included in Laigle et al. (2016), we derived it in the following way. For each source in the sample, we reconstructed the galaxy SED using Hyperzmass, which is a modified version of the Hyperz software (Bolzonella et al. 2000, 2010) and is suitable for computing the stellar mass when the photometric redshift is known. We adopted the Bruzual \& Charlot (2003) stellar population models, with exponentially declining SFH, that is, $\left.\mathrm{SFR} \propto e^{t / \tau}\right)$, with $\tau=0.1,0.3,1,2,3,5,10,15,30, \infty=\mathrm{constant}$ SFR). The $L_{1600 \AA}$ were extracted directly from the Hyperzmass best-fit templates. The SFRs for quiescent and star-forming galaxies are shown in the upper left and central panels of Fig. 3 as a function of stellar mass. They are color-coded based on redshift.

For starburst galaxies we used the $S_{F R}$ from Gruppioni et al. (2013) (see Sect. 2.2) after verifying that their $\mathrm{SFR}_{\mathrm{UV}}$ is negligible. Similarly to star-forming and quiescent galaxies, we performed bootstrapping for every $M_{*}$ and $z$ bin, where in each loop we derived a median $\mathrm{SFR}_{\mathrm{IR}}$ and therefore an $\mathrm{SFR}_{\mathrm{IR}}$ distribution. The SFRs for starburst galaxies are shown in the top right panel of Fig. 3.

\section{Comparing the SMBH X-ray emission at the different galaxy life stages throughout cosmic time}

In Fig. 2 we show the X-ray $2-10 \mathrm{keV}$ luminosity $\left(L_{X}\right)$ from Eq. (3) as a function of stellar mass for our sample, divided into different redshift bins. For each bin we compared the X-ray luminosity for the three types of galaxies: star-forming, quiescent, and starbursts. Our data points are centered on the median Xray luminosity and stellar mass. Vertical error bars are the $90 \%$ confidence range. We show a log-log linear fit for star-forming galaxy data points. The fits were performed ignoring the upper limit data points.

We find a robust relation between the average $L_{X}$ and $M_{*}$ in star-forming galaxies: this indicates that black holes in more massive galaxies grow faster than black holes in the less massive galaxies. This might in part be sustained by the higher fraction of type I AGN at high stellar masses, as seen by Suh et al. (2019) for Chandra COSMOS Legacy detected sources. Moreover, this relation increases in normalization by about $1.5 \mathrm{dex}$ but maintains an almost constant slope of about $\sim 1.00$ toward higher redshift, which indicates that black holes grew faster at earlier epochs than they do today. This is shown in Table 2, which lists the best linear fit to our data $\left(\log L_{X}=a \log M_{*}+b\right)$. This relation looks very similar to the main-sequence of star-forming galaxies, which is the relation between SFR and $M_{*}$, as we show more clearly in Fig. 3. The quiescent $24 \mu \mathrm{m}$ MIPS/Spitzer detected sources are a small percentage of the overall star-forming sample $(\sim 2 \%)$, but they are mostly concentrated at the high masses and low redshifts, where they reach a percentage as high as $86 \%$ at $M_{*}>10^{11} M_{\odot}$ in the lowest redshift. The addition of these sources had very little effect on the $L_{X}$ of the higher mass bins, but all bins show a systematical increase in $L_{X}$ of $\lesssim 0.1$ dex.

Starburst galaxies show average $L_{X}$ with a similar dependence on stellar mass as star-forming galaxies and a mild dependence on redshift: as a result, the X-ray luminosities of starbursts are $\sim 0.4-1.1$ dex higher than those of star-forming galaxies at $z \simeq 0.4$, but the luminosities at $z \simeq 2-3$ are compatible. If these galaxies undergo a major merger event, as is commonly believed, this suggests that while a higher availability of cold gas allows the SFR to increase considerably with respect to a galaxy in a secular evolution phase, this gas availability is not able to accrete onto the black hole at a pace higher than a certain threshold that does not vary as much as the star-forming population $(\sim 0.5 \mathrm{dex})$ in the redshift range we covered.

Finally, quiescent galaxies have X-ray luminosities that tend to be lower than those of star-forming galaxies. They vary with increasing mass from $\sim 0.5$ dex to $\sim 1$ dex in difference, with the exception of the highest redshift bin, which is almost compatible with the luminosity of main-sequence galaxies. The resulting relation with stellar mass is flatter than the relation observed for star-forming galaxies. The relation is limited to just a few (high-) mass bins because in the mass regime below $10^{10} M_{\odot}$, the 


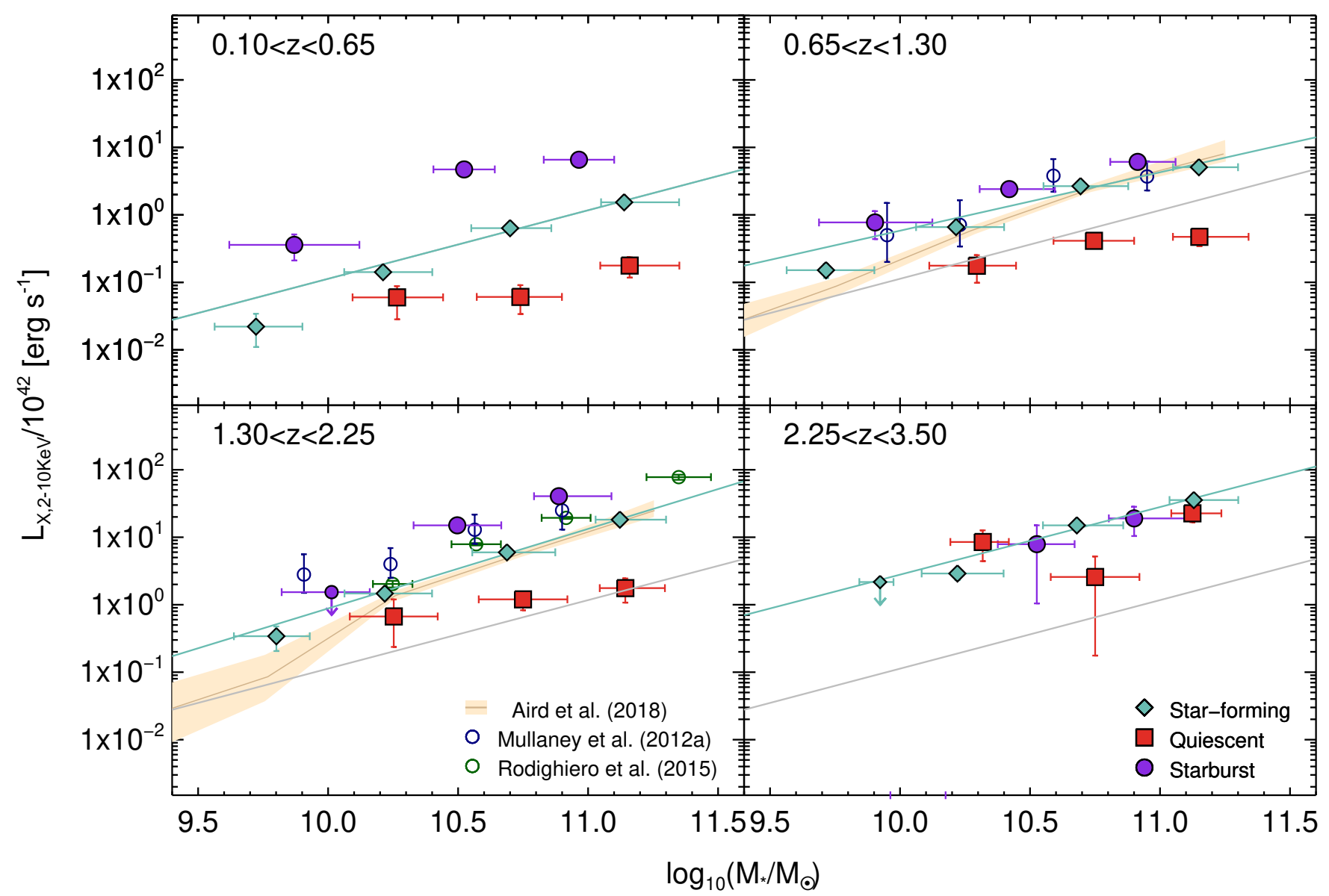

Fig. 2. X-ray luminosity in the $2-10 \mathrm{keV}$ band for our sample as a function of stellar mass. Each panel represents one of the four redshift bins that we considered. The light green diamonds represent the normal star-forming sample, the red squares represent the quiescent sample, and the violet circles represent the starburst sample. The data points represent the median value of the stellar mass and the X-ray luminosity. Vertical error bars represent the 5th and 95th percentile (90\% confidence interval) of the distributions, while horizontal error bars correspond to $1 \sigma$. We show an upper limit (the 95th percentile) for the data points whose X-ray luminosity 5th percentile is compatible with zero and for starburst galaxies at high redshift and low mass, where our sample is incomplete. The light green continuous line is the best fit for the star-forming galaxy data points at each redshift, while the gray line shows the best fit in the lowest redshift bin, shown for comparison purposes. We also report data points from Mullaney et al. (2012b) at an average redshift of $z=1,2$ (open dark blue circles, Rodighiero et al. 2015) at an average redshift of $z=2$ (open green circles) and Aird et al. (2018) at $z=1,2$ (solid brown curve; the yellow area shows the $1 \sigma$ confidence interval). The data points from Rodighiero et al. (2015) and Aird et al. (2018) are scaled to the same $k$-correction as we adopted here.

Table 2. X-ray luminosity fits to the equation $\log _{10} L_{X}=a \log _{10} M_{*}+b$ for the star-forming galaxies of our sample.

\begin{tabular}{lcc}
\hline \hline Redshift bin & $a$ & $b$ \\
\hline $0.10<z<0.65$ & $1.02 \pm 0.03$ & $-11.1 \pm 0.3$ \\
$0.65<z<1.30$ & $0.87 \pm 0.02$ & $-8.9 \pm 0.2$ \\
$1.30<z<2.25$ & $1.18 \pm 0.03$ & $-11.8 \pm 0.3$ \\
$2.25<z<3.50$ & $1.00 \pm 0.05$ & $-9.6 \pm 0.5$ \\
\hline
\end{tabular}

Notes. Errors are $1 \sigma$ uncertainty estimates of parameters.

X-ray flux was not high enough to constrain the X-ray luminosity of the subsample: we obtained a 95th percentile luminosity value $<0$. According to the hardness ratio analysis performed by Paggi et al. (2016) on a subsample from C-COSMOS of earlytype stacked galaxies, the X-ray luminosities of our quiescent galaxies are expected to be compatible with a combination of thermal and AGN emission in the lower two redshift bins and with highly obscured AGN at higher redshifts. This confirms the origin of the emission.
In Fig. 2 we compare our results for the sample of star-forming galaxies with literature results by Mullaney et al. (2012b), Rodighiero et al. (2015), and Aird et al. (2018) after scaling the luminosity to the same $k$-correction as we used in our analysis where necessary (i.e., rescaled to the same photon index $\Gamma$ in the $k$-correction). At $z \sim 1$, our data points agree well with those of Mullaney et al. (2012b) and Aird et al. (2018). At $z \sim 2$ our results are in great agreement, within the errors, with the results of Aird et al. (2018), while our X-ray luminosities are systematically lower than those by Mullaney et al. (2012b) and Rodighiero et al. (2015).

\section{Constraining the coevolution of galaxy and black hole accretion}

In Fig. 3 we compare the SFR and the BHAR as a function of $M_{*}$ for the three categories of galaxies. In the left column we show star-forming galaxies, in the middle column we list quiescent galaxies, and the right column contains starburst galaxies. The top panel shows the total $\mathrm{SFR}_{\mathrm{UV}+\mathrm{IR}}\left(\mathrm{SFR}_{\mathrm{IR}}\right.$ only for starburst galaxies, see Sect. 4.3) for all the redshift bins, the middle panel 


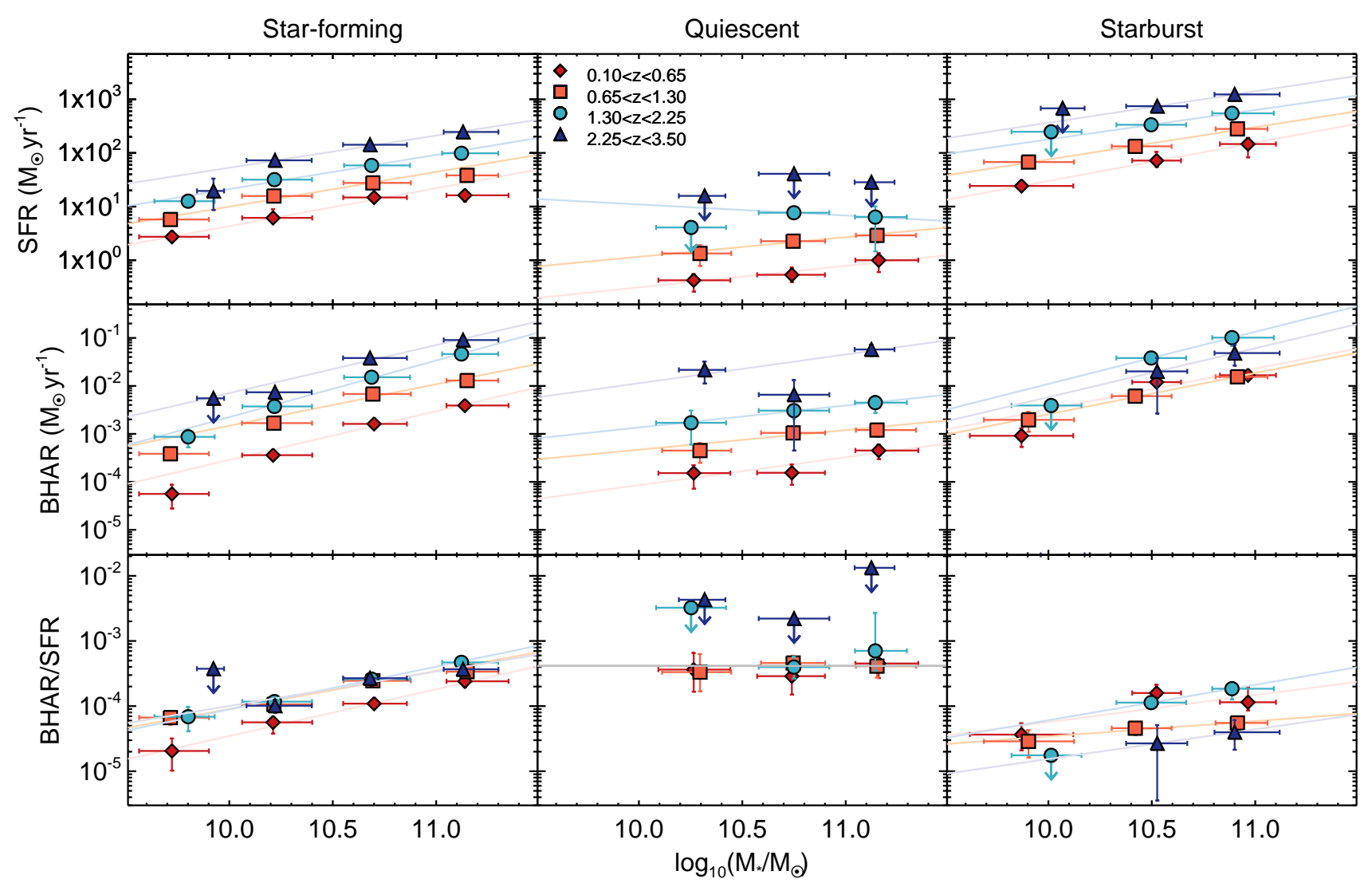

Fig. 3. Comparison between the average SFR and the average BHAR for the three samples: normal star-forming galaxies (left), quiescent galaxies (center), and starburst galaxies (right). In all plots data points are color- and shape-coded according to the redshift interval. The data points are the median value, and vertical errors represent the 5th and 95th percentile. We show an upper limit (the 95th percentile) for the data points whose SFR or BHAR 5th percentile is compatible with zero and for starburst galaxies at high redshift and low mass, where our sample is inclomplete in mass. The error bars shown on the stellar masses correspond to $1 \sigma$ of the distribution. The solid light colored lines are the best fits and are color-coded according to each redshift bin (see Table 3). Top panel: $M_{*}$-SFR relation. Middle panel: $M_{*}$-BHAR relation. Bottom panel: BHAR-to-SFR ratio as a function of stellar mass. The dotted gray line for quiescent galaxies is at a constant value of BHAR/SFR $=3.8 \times 10^{-4}$.

shows the BHAR of the sample obtained from the X-ray luminosity (Fig. 2) using Eq. (5), and the bottom panel presents the ratio between BHAR and SFR. We show linear fits in all panels except for the BHAR/SFR of quiescent galaxies where we report the average value of all redshifts and mass bins because no evolutionary trends are apparently visible. The best-fit parameters are shown in Table 3.

\subsection{Star-forming galaxies}

For star-forming galaxies, the top panel shows the well-known evolution of the main-sequence of star-forming galaxies with time: the SFR follows a sublinear relation (in logaritmic units) with stellar mass that evolves in normalization with redshift, maintaining an almost constant slope and a bend at high $M_{*}$ in the two lower redshift bins. This bend was introduced by including the quiescent $24 \mu \mathrm{m}$ MIPS/Spitzer detected sources, which as described above, are concentrated at high masses and lower redshifts. The median $\mathrm{SFR}_{\mathrm{UV}}$ of the sample is lower than the median $\mathrm{SFR}_{\mathrm{IR}}$ by a factor that varies from 3 (low mass) to 100 (high mass, see Table A.1), which means that the $\mathrm{SFR}_{U V}$ is negligible in most cases.

The observed BHAR distribution has a higher slope in the $\log -\log$ plane; it is slightly superlinear (a direct consequence of the $L_{X}-M_{*}$ relation). It is present at all considered redshifts and evolves with it. We note that the decrease in BHAR normalization evolves faster in the lowest redshift bin, but maintains a more constant increase at earlier cosmic epochs. The total decrease in BHAR at given $M_{*}$ is about $1.5 \mathrm{dex}$, and in SFR, the decrease is about $1-1.2$ dex.

The ratio of BHAR to SFR provides interesting insights into how these two phenomena relate to each other. It increases with mass with a slope that varies in the $\sim 0.5-0.7$ range, decreases at higher redshifts, and does not evolve in normalization. The positive slope of the BHAR/SFR can be interpreted as an indication of an increased accretion efficiency in galaxies with high stellar mass by driving the infalling gas directly toward their inner core, which fuels a faster accretion onto the black hole. This can also be considered to mean that the main driver of the black hole accretion is not time, but the initial stellar mass. We note that the analogy between the increase in the BHAR/SFR as a function of stellar mass and the increase in the density of the central kiloparsec of the galaxy $\Sigma_{1}$ with mass (Fang et al. 2013; Barro et al. 2017). It has been proposed that more massive galaxies might have denser cores that might allow for a faster growth of the black hole.

\subsection{Quiescent galaxies}

In the central column of Fig. 3 we show the data points of the quiescent galaxies. As expected, the SFR of these galaxies is lower than that of star-forming galaxies. The two lower redshifts 
Table 3. Fit parameters of the relations in Fig. 3.

\begin{tabular}{|c|c|c|c|c|c|c|}
\hline \multirow[b]{2}{*}{ Redshift bin } & \multicolumn{2}{|c|}{ SFR } & \multicolumn{2}{|c|}{ BHAR } & \multicolumn{2}{|c|}{ BHAR/SFR } \\
\hline & $m$ & $q$ & $m$ & $q$ & $\alpha$ & $\beta$ \\
\hline \multicolumn{7}{|l|}{ Star-forming } \\
\hline $0.10<z<0.65$ & $0.71 \pm 0.02$ & $-6.4 \pm 0.2$ & $1.02 \pm 0.03$ & $-13.7 \pm 0.3$ & $0.72 \pm 0.09$ & $-11.6 \pm 0.9$ \\
\hline $0.65<z<1.30$ & $0.649 \pm 0.013$ & $-5.49 \pm 0.13$ & $0.87 \pm 0.02$ & $-11.5 \pm 0.2$ & $0.58 \pm 0.03$ & $-9.9 \pm 0.4$ \\
\hline $1.30<z<2.25$ & $0.639 \pm 0.018$ & $-5.06 \pm 0.19$ & $1.18 \pm 0.03$ & $-14.4 \pm 0.3$ & $0.65 \pm 0.04$ & $-10.6 \pm 0.4$ \\
\hline $2.25<z<3.50$ & $0.60 \pm 0.04$ & $-4.3 \pm 0.4$ & $1.00 \pm 0.05$ & $-12.2 \pm 0.5$ & $0.53 \pm 0.07$ & $-9.3 \pm 0.7$ \\
\hline \multicolumn{7}{|l|}{ Quiescent } \\
\hline $0.10<z<0.65$ & $0.40 \pm 0.14$ & $-4.5 \pm 1.5$ & $0.58 \pm 0.17$ & $-9.9 \pm 1.9$ & & \\
\hline $0.65<z<1.30$ & $0.37 \pm 0.15$ & $-3.6 \pm 1.6$ & $0.41 \pm 0.16$ & $-7.4 \pm 1.7$ & - & $-3.38 \pm 0.07$ \\
\hline $1.30<z<2.25$ & $-0.2 \pm 0.5$ & $3.1 \pm 4.9$ & $0.5 \pm 0.2$ & $-7.6 \pm 2.4$ & & \\
\hline $2.25<z<3.50$ & - & - & $0.59 \pm 0.18$ & $-7.9 \pm 2.0$ & & \\
\hline \multicolumn{7}{|l|}{ Starburst } \\
\hline $0.10<z<0.65$ & $0.73 \pm 0.05$ & $-5.8 \pm 0.5$ & $0.86 \pm 0.09$ & $-11.0 \pm 1.0$ & $0.36 \pm 0.12$ & $-7.9 \pm 1.3$ \\
\hline $0.65<z<1.30$ & $0.61 \pm 0.03$ & $-4.3 \pm 0.3$ & $0.86 \pm 0.10$ & $-11.1 \pm 1.1$ & $0.22 \pm 0.11$ & $-6.7 \pm 1.2$ \\
\hline $1.30<z<2.25$ & $0.54 \pm 0.07$ & $-3.1 \pm 0.8$ & $1.08 \pm 0.11$ & $-12.8 \pm 1.2$ & $0.54 \pm 0.15$ & $-9.6 \pm 1.6$ \\
\hline $2.25<z<3.50$ & $0.59 \pm 0.14$ & $-3.4 \pm 1.5$ & $1.0 \pm 0.7$ & $-12.5 \pm 7.3$ & $0.44 \pm 0.72$ & $-9.2 \pm 7.8$ \\
\hline
\end{tabular}

Notes. The equations we used are for $\log _{10} \mathrm{SFR}=m \log _{10} M_{*}+q$; for $\log _{10} \mathrm{BHAR}=m \log _{10} M_{*}+q$; and for the ratio $\log \frac{\text { BHAR }}{\mathrm{SFR}}=\alpha \log M_{*}+\beta$.

show a weak dependence of stellar mass, with a slope of $\sim 0.4$, and the evolution with redshift in the bins in which we were able to constrain the SFR is also clear. The reason might be that high-redshift elliptical galaxies are of a different nature than local galaxies and have higher percentages of gas (Gobat et al. 2018), but part of the emission might also originate in dust cirrus illuminated by older stellar populations (Draine \& Li 2007; Béthermin et al. 2015). Another factor that may be contributing to the evolution we see is a small fraction of misclassified star-forming galaxies at high redshift. This may be caused by galaxies that emit in the MIPS band but lie below the detection threshold or by cross-contamination in the NUV $-r / r-J$ because the uncertainty in the estimation of the rest-frame magnitudes that are required to classify a galaxy in the color-color diagram is higher. Overall, it is not possible to determine whether this evolution in time and mass dependence of quiescent galaxies and their mass dependence is significant, especially because we are able to constrain only these high-mass bins.

For comparison with star-forming galaxies, we note that the median $S_{F} R_{U V}$ of the quiescent sample is lower by a factor of about 15 than the median SFR $_{I R}$ (see Table A.2). This means that the $\mathrm{SFR}_{\mathrm{UV}}$ is negligible in most cases.

We are better able to constrain the BHAR at all redshifts and see a redshift evolution and weak dependence on stellar mass, with a slope $\sim 0.5$, even though it is difficult to analyze the slope evolution because of the small number of data points. The normalization in BHAR is higher than in SFR, that is, the normalization of the BHAR is similar to the normalization of BHAR in star-forming galaxies, while the SFR of quiescents is clearly lower than that of star-forming galaxies. This indicates that the efficiency in accreting material onto the black hole is higher than in forming stars in galaxies at this late stage in the life of galaxies: the gas present in the galaxy "prefers" to fall into the black hole rather than form stars. This is consistent with the results of Gobat et al. (2018), who reported that a substantial amount of gas is available at high redshift in quiescent galaxies. This is then probably consumed less efficiently than in star-forming galaxies.

The BHAR/SFR of quiescent galaxies, for the few mass and redshift bins where it was possible to constrain this, shows a flat trend in mass that is compatible with a constant value of
$4.2 \times 10^{-4}$ at all redshifts and $M_{*}$. This value, obtained as a weighted mean of the data points, is a confirmation of the trends we have seen for BHAR and SFR: it is compatible with the values obtained for the highest mass bin of star-forming galaxies, indicating that even though the ability of a galaxy to form stars has decreased in these galaxies, the efficiency of attracting the gas to the black hole did not.

\subsection{Starburst galaxies}

In the right column of Fig. 3 we show the starburst sample. By selection, FIR-selected starbursts have higher SFR than normal star-forming galaxies; the evolution in time is clear, as is the dependence on $M_{*}$.

In starburst galaxies, the BHAR shows a close to linear dependence on mass and a weak evolution in redshift: values range in about 1 dex, but the highest redshift bin has a lower normalization than the previous one, differently from what is seen in the other phases of galaxy life. This decrease in BHAR, which is not as pronounced as the decrease in star-forming galaxies (about $\sim 1.5 \mathrm{dex}$ ) causes them to slowly become starburst galaxies (i.e., higher than main-sequence galaxies) in the black hole accretion as well at lower redshift.

There is no clear evolution in the normalization of the BHAR/SFR, but it is interesting to note that the highest redshift bin shows the lowest normalization, which indicates that in these extremely star-forming galaxies black hole accretion was disfavored at higher redshifts. The slope appears to be positive and of about $0.2-0.4$.

We tested selecting a starburst galaxy sample using the mainsequence from Rodighiero et al. (2011) with a redshift evolution as in Sargent et al. (2012), $\operatorname{SFR}_{\mathrm{MS}}(z) \propto(1+z)^{2.8}$. This selection assumed no bend on the main-sequence at high stellar masses and was calibrated up to $z \sim 2$. When we extrapolated this evolution to higher redshifts, we found a higher normalization than was reported by Schreiber et al. (2015), which instead was calibrated up to $z \sim 5$. This starburst selection led to no significant differences in the SFR of the sample, but to slightly lower BHAR values. We were also unable to constrain the X-ray luminosities of starbursts in the highest redshift bin. This suggests that the 
most starbursty galaxies accrete their black hole even less. This finally resulted in a flatter trend of the BHAR/SFR against stellar mass.

\subsection{Comparisons with the literature}

In Fig. 4 we compare our results for the star-forming sample with results from the literature. We show our data points from Fig. 3 in the left column and compare them with results from Yang et al. (2018) as solid lines, with results from Aird et al. (2019) as a dashed beige line, and with results from Delvecchio et al. (2019) as a dot-dashed violet line.

Our data points qualitatively agree with those from Yang et al. (2018) in the three panels . Yang et al. (2018) selected their star-forming sample from GOODS-N, GOODS-S, and COSMOS UltraVISTA DR1 based on the SFR from SED fitting with a SFR threshold at 1.3 dex below the main-sequence. SFRs are from Santini et al. (2015) and Barro et al. (2019). Their average SFRs are slightly higher than ours. They also reported that the BHAR of the lowest redshift bin is separated more than the BHAR between the other bins.

The BHAR/SFR of Aird et al. (2019) has a linear trend with mass with a higher slope than ours. Their BHARs were obtained with the same method as in Aird et al. (2018). Their SFRs are from SED fitting from the UV to MIR, which means that the FIR was not included. Together with the constant bolometric correction they used, this might lead to the higher slope value.

We also compared our data points with the relation found by Delvecchio et al. (2019) through an empirically motivated model that successfully reproduces the observed X-ray luminosity function (XLF) since $z \sim 3$. With this model, they found a growth in two steps: until the galaxy reaches a critical mass, the black hole growth lags behind it, and then, as the stellar mass increases, the BHAR is enhanced with respect to the SFR, following a superlinear relation very similar to ours, except for an offset of $\sim 0.1-0.3 \mathrm{dex}$.

\section{Comparison between the evolution of SBHAR and SSFR}

We computed the average sSFR (i.e., specific SFR, SFR $/ M_{*}$ ) and the sBHAR (i.e., specific BHAR, BHAR/ $M_{\mathrm{BH}}$ ) for all the bins in $M_{*}$ and $z$. In this section we describe how the sBHAR was derived and we discuss the results.

\subsection{Black hole mass estimate}

In order to estimate the black hole mass $M_{\mathrm{BH}}$, we considered the fits in the bottom panels of Fig. 3 and in the right column of Table 3, obtained from the equation

$\log \frac{\mathrm{BHAR}}{\mathrm{SFR}}=\alpha \log M_{*}+\beta$.

The general equation we used for the computation is

$\log \frac{\mathrm{BHAR}}{\mathrm{SFR}}=\log \frac{\dot{M}_{\mathrm{BH}}}{\dot{M}_{*}}=\log \frac{\partial M_{\mathrm{BH}}}{\partial M_{*}}=\alpha \log M_{*}+\beta$,

which is valid only under the assumption that the growth rates of stellar and black hole mass are constant in time. The ratios we measure show almost no evolution up to $z \sim 3.5$, which appears to justify this assumption. By integrating Eq. (8) with respect to

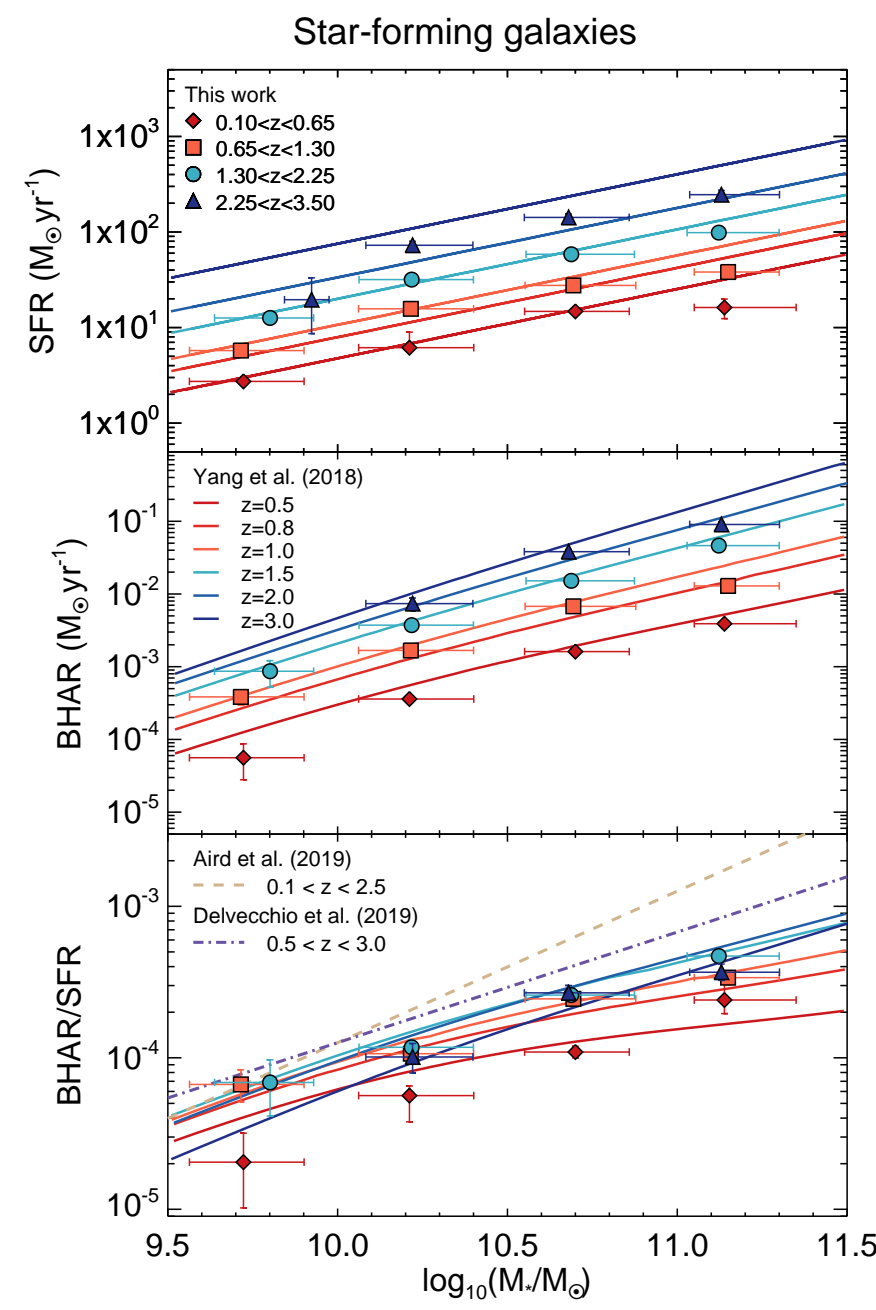

Fig. 4. Comparison between our results for star-forming galaxies and data from Yang et al. (2018), Aird et al. (2019), and Delvecchio et al. (2019). Data points are taken from the left column of Fig. 3, and the curves are adapted from Fig. 14 in Yang et al. (2018) and were scaled to the same $k$-correction as we adopted here. Data from Aird et al. (2019) are taken from Fig. 13.

stellar mass, we obtained the estimate of the $M_{\mathrm{BH}}$ of each stellar mass and redshift bin:

$M_{\mathrm{BH}}=\frac{10^{\beta}}{1+\alpha} M_{*}^{1+\alpha}$,

where $\alpha$ and $\beta$ are the same parameters in Table 3 and are the parameters we used in the computation. It follows from Eq. (9) that the black hole mass has a superlinear dependence on stellar mass in star-forming and starburst galaxies, but $M_{\mathrm{BH}}$ has a linear dependence on $M_{*}$ in quiescent galaxies.

If instead of performing an indefinite integral we integrate between the initial masses $\left(M_{*, i}\right.$ and $\left.M_{\mathrm{BH}, i}\right)$ and the final (observed) masses, the integrated Eq. (8) would read

$M_{\mathrm{BH}}=\frac{10^{\beta}}{1+\alpha}\left(M_{*}^{1+\alpha}-M_{*, i}^{1+\alpha}\right)+M_{\mathrm{BH}, i}$.

We assumed that the initial black hole mass is lower by at least an order of magnitude $\left(\lesssim 10^{5} M_{*}\right)$ than the final mass, however, therefore it is negligible. The initial stellar mass instead eludes us, and becuase we subtracted it, our $M_{\mathrm{BH}}$ is an upper limit. We therefore decided not to use the complete expression of $M_{\mathrm{BH}}$ in Eq. (10), but the expression in Eq. (9). 


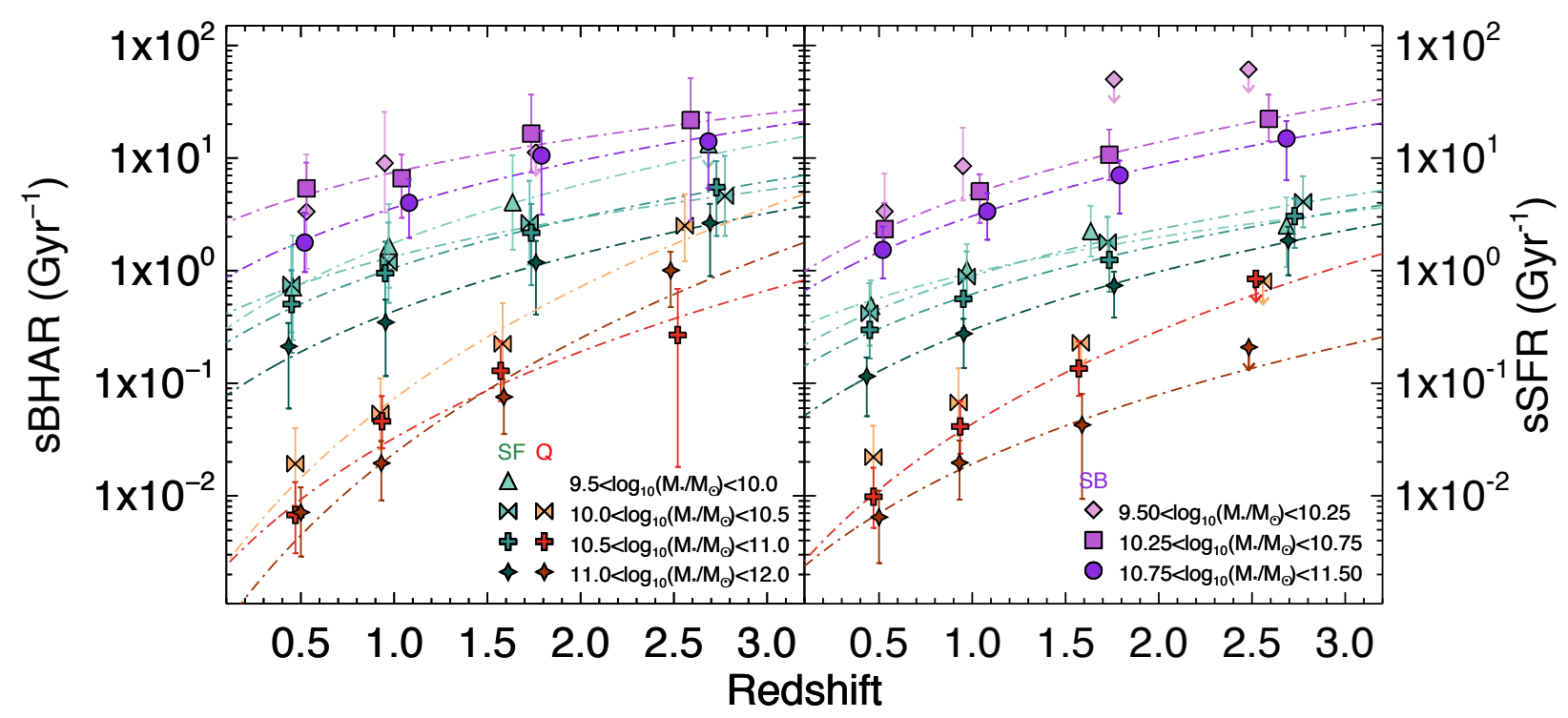

Fig. 5. Specific black hole accretion rate (left panel) and sSFR (right panel) as a function of redshift for star-forming (SF, in green), quiescent (Q, in red), and starburst (SB, in purple) galaxies. The data points are placed at the median redshift of each mass bin and are coded in shape and color according to their median mass and type. Error bars represent the $90 \%$ confidence interval associated with each measure. We show an upper limit (the 95th percentile) for the data points whose sBHAR/sSFR 5th percentile is compatible with zero and for starburst galaxies at high redshift and low mass, where our sample is incomplete. The dot-dashed line is the best fit of the data to the curve $\operatorname{sBHAR}(\operatorname{sSFR})=\delta(1+z)^{\gamma}$ and is color-coded according to the galaxy type and mass bin.

\subsection{Results for the specific accretions}

Figure 5 shows the redshift evolution of the sBHAR (estimated using $M_{\mathrm{BH}}$ from Eq. (9)) and sSFR for star-forming, quiescent, and starburst galaxies. sBHAR and sSFR have a decreasing trend toward lower redshift for the three galaxy types, and the stellar mass shows a split: low-mass galaxies have higher values than high-mass galaxies, although this split is often not significant within the error bars.

These trends confirm what has been reported before about these specific accretions. They also provide new interesting insight. The spread in stellar mass is consistent with downsizing: it implies that high-mass galaxies have accreted most of their $M_{*}$ and $M_{\mathrm{BH}}$ at high redshift and their accretion decreased fast and steeply, whereas low-mass galaxies have accreted their mass more slowly, but their accretion rate decreased more slowly with time. Downsizing has previously been observed for stellar mass (Cowie et al. 1996; Cimatti et al. 2006) and black hole luminosity (e.g., Marconi et al. 2004 but also Shankar et al. 2004, 2009; Aversa et al. 2015, who reached similar conclusions from continuity equation arguments). In our plots, downsizing can be observed in the steepening of the slope $\gamma$ at higher masses in star-forming and starburst galaxies and in the two specific accretions, which indicate a faster decrease for higher masses. Downsizing can also be seen in high-mass galaxies (darker data points), which show lower sSFR(sBHAR) on average than lower mass galaxies. This means that at even at higher redshifts, the most massive galaxies have already accreted most of their stellar and black hole mass, even though within the error bars, sSFR (sBHAR) data points are often compatible with a unique value for all stellar masses at a given redshift. In particular, this trend would not be present in star-forming galaxies without introducing the dependence of the black hole mass on the slope $\alpha$ from Eq. (7).

We show in Fig. 5 the best fits to the data when a redshift evolution of the form sBHAR (sSFR) $=\delta(1-z)^{\gamma}$ is adopted, which we applied to each mass bin for all galaxy types when at least three data points were available ${ }^{3}$. The sSFR and sBHAR for star-forming and starburst galaxies are compatible with $\gamma=2.8$, as in Sargent et al. (2012), within $1 \sigma$, but this is not the case for quiescent galaxies, which have higher slopes with a steeper decreases in specific accretions in time. Interestingly, we do not note a significant exponent difference between SBHAR and sSFR at given galaxy type. When we consider that there may be a small contribution from misclassified star-forming galaxies in the quiescent sample, especially at high redshift, the real trend may be even flatter, and approach a slope $\gamma=2.8$. We do see a difference in the normalizations of the relations, which show lower specific accretions for quiescent galaxies, followed by star-forming galaxies, and finally, starburst galaxies, which tend to have higher specific accretions. When the normalizations for the two specific accretions are compared, they are very similar in the case of starburst galaxies, while star-forming galaxies have a higher normalization for the sBHAR and quiescent galaxies show higher sBHAR than SSFR at high $z$.

Because the inverse of sSFR and sBHAR can be considered as the mass-doubling timescale of the $M_{*}$ and of the SMBH, this means that the stellar mass of the galaxy and the SMBH are accreted faster in starburst and star-forming galaxies, which especially at high redshift are still efficient and can quickly double their mass. Quiescent galaxies are instead slower and have mass-doubling timescales of about a Hubble time or more. The black hole mass-doubling time of star-forming galaxies instead seems to be shorter than the stellar mass black hole massdoubling time at every redshift and mass bin.

These similar evolution trends between SBHAR and SSFR strongly suggest a connection between the two accretions that appears to be present in all galaxy life phases. Star-forming galaxies appear to dominate the accretion histories; they are the most numerous galaxies and are able to substantially accrete their stellar and black hole masses. Starburst galaxies, with a higher capability of accretion but short-lived episodes, and

3 Fits performed with IDL/MPFIT (Markwardt et al. 2009). 


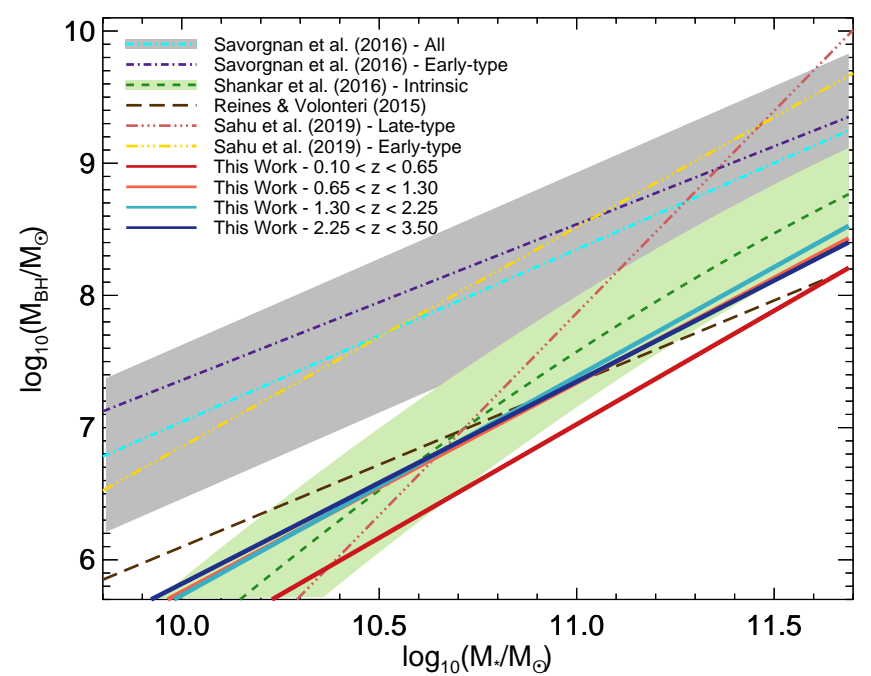

Fig. 6. Comparison between our $M_{*}$ and $M_{\mathrm{BH}}$ from Eq. (9) and parameters $\alpha$ and $\beta$ from Table 3 for star-forming galaxies and results from models in the literature. Data from this work are shown as solid lines and are color-coded according to redshift as in Figs. 3 and 4. The relations from Savorgnan \& Graham (2016) use the $M_{*}$ of the bulge. The gray shaded area is the confidence range for Savorgnan \& Graham (2016), and the light green are is the confidence range for Shankar et al. (2016).

quiescent galaxies, although their accretion capabilities are lower, appear to be able to accrete their black hole more efficiently than their stellar mass.

\section{Relation between stellar mass and black hole mass}

In Fig. 6 we show our best-fit resulting $M_{\mathrm{BH}}-M_{*}$ relations for star-forming galaxies derived from Eq. (9) with parameters from Table 3. These are superlinear relations with approximately $M_{\mathrm{BH}} \propto M_{*}^{1.6}$. Our stellar masses are to be considered lower limits because as pointed out in Bell et al. (2003), stellar masses obtained using Bruzual \& Charlot (2003) models lead to lower mass-to-light ratios and therefore to systematically lower stellar mass estimates at a given luminosity. A possible correction in our stellar masses that would cause them to agree better with those of Bell et al. (2003) (increase of $\sim 0.25$ dex) would further support our results.

We note that recent scaling relations proposed by other groups for both early-type galaxies and late-type galaxies show different scaling slopes and normalizations. Sahu et al. (2019) find a relation for early-type galaxies that is similar in both slope and normalization to the lines of Savorgnan \& Graham (who used bulge stellar masses. See Shankar et al. (2016, 2019) for details) reported in Fig. 6. For late-type galaxies, Sahu et al. (2019) find a lower normalization, more consistent with our relation around $M_{*} \sim 10^{10.5} M_{\odot}$, but a much steeper slope of $\sim 3.0 \pm 0.5$, about $3 \sigma$ steeper than ours. All in all our results point to either lower normalizations or flatter slopes than those identified from local dynamically measured SMBHs. On the other hand, our resulting scaling relations have a similar normalization with those proposed by Reines \& Volonteri (2015) from local broad line AGN, despite them finding a close to linear relation, and agree well with the intrinsic scaling relation by Shankar et al. (2016). The latter have suggested that the local sample of (mainly) early-type galaxies with dynamically measured SMBH may be biased because a preselection might favor galaxies with the highest black hole masses and related gravitational radii. As recently suggested by Shankar et al. (2019), AGN samples that are clearly not affected by resolutionrelated selections effects should be closer to the intrinsic scaling relations. Our study further supports this view.

We note that our normalization is inversely proportional to the radiative efficiency assumed in Eq. (5). A match to the normalization of the local $M_{\mathrm{BH}}-M_{*}$ relation by Savorgnan \& Graham would require mean radiative efficiencies that are an order of magnitude lower. This is disfavored on other grounds (Shankar et al. 2020).

Our analysis adds key evidence, using IR data, to an increasing body of work (Delvecchio et al. 2019; Ding et al. 2020; Shankar et al. 2020; Suh et al. 2020) on the weak evolution in the BHAR/SFR and its relatively low normalization relative to local raw dynamical $M_{\mathrm{BH}}-M_{*}$ relations.

\section{Conclusions}

We performed a statistical study on the COSMOS field in order to constrain the history and coevolution of star formation and black hole accretion in star-forming, quiescent, and starburst galaxies. We selected a mass-complete sample from the COSMOS 2015 catalog (Laigle et al. 2016) by classifying normal star-forming and quiescent galaxies through the NUV $-r / r-J$ color-color diagram, and we selected starburst galaxies from the Herschel-selected sample in Gruppioni et al. (2013). We performed an X-ray stacking analysis and combined it with detected sources from Civano et al. (2016) in order to estimate the average X-ray luminosity and therefore average BHAR. We estimated the SFR from FIR stacking and UV SED fitting. Our main results are listed below.

1. We find a robust $L_{X}-M_{*}$ relation for star-forming galaxies at all considered redshifts. This relation evolves with an increasing normalization at higher redshifts. The X-ray luminosity of quiescent galaxies is close to that of star-forming galaxies, especially at low masses. At high masses, $L_{X}$ in quiescent galaxies is lower than in star-forming galaxies. The X-ray luminosities of starburst galaxies are compatible with star-forming at high redshifts and evolve mildly down to low redshift, where they are clearly higher than those of starforming galaxies.

2. The $L_{X}-M_{*}$ relation translates into a BHAR- $M_{*}$ relation in Fig. 3 (middle row) that shows that the evolution of the BHAR in star-forming galaxies is faster at lower redshifts, it has a pronounced redshift evolution, and a weak mass dependence in quiescent galaxies. In turn, starburst galaxies have a marked mass dependence and a distinctive redshift evolution: going back in time, it reaches a maximum at $z \sim 1.7$ to then decrease again.

3. BHAR in star-forming galaxies increases more with stellar mass than the SFR. In quiescent galaxies, the BHAR values lie close to the BHAR of star-forming galaxies, while the SFR of quiescent galaxies is clearly below the mainsequence. It is interesting that while the SFR of starburst galaxies continues to increase at higher redshifts, at the highest redshift in our study, the BHAR of these galaxies decreases.

4. The ratio between BHAR and SFR in star-forming and starburst galaxies has a positive relation $M_{*}$ that is almost timeindependent. The ratio is higher for quiescent galaxies, compatible with a flat trend in $M_{*}$, indicating a stronger tendency for this type of galaxy to accrete onto the black hole than 
Table 4. Fit parameters of the relations in Fig. 5.

\begin{tabular}{lccccc}
\hline \hline Mass bins & \multicolumn{2}{c}{ sBHAR parameters } & & \multicolumn{2}{c}{ sSFR parameters } \\
\cline { 2 - 3 } \cline { 5 - 6 } $\log _{10}\left(M_{*} / M_{\odot}\right)$ & $\delta$ & $\gamma$ & & $\delta$ & $\gamma$ \\
\hline Star-forming & & & & \\
$9.5-10.0$ & $0.2 \pm 0.3$ & $2.9 \pm 1.8$ & & $0.28 \pm 0.13$ & $1.8 \pm 0.5$ \\
$10.0-10.5$ & $0.3 \pm 0.3$ & $2.0 \pm 0.9$ & & $0.17 \pm 0.09$ & $2.4 \pm 0.6$ \\
$10.5-11.0$ & $0.18 \pm 0.14$ & $2.6 \pm 0.8$ & & $0.11 \pm 0.05$ & $2.5 \pm 0.5$ \\
$11.0-12.0$ & $0.06 \pm 0.04$ & $2.9 \pm 0.7$ & & $0.039 \pm 0.016$ & $2.9 \pm 0.4$ \\
\hline Quiescent & & & & - \\
$10.0-10.5$ & $0.0015 \pm 0.0012$ & $5.6 \pm 0.9$ & & - & \\
$10.5-11.0$ & $0.0016 \pm 0.0009$ & $4.4 \pm 0.7$ & & $0.0017 \pm 0.0012$ & $4.7 \pm 1.0$ \\
$11.0-12.0$ & $0.0004 \pm 0.0003$ & $5.8 \pm 0.8$ & & $0.0017 \pm 0.0013$ & $3.5 \pm 1.1$ \\
\hline Starburst & & & & & - \\
$9.50-10.25$ & - & - & & - & $2.6 \pm 0.5$ \\
$10.25-10.75$ & $2.3 \pm 1.6$ & $1.7 \pm 0.9$ & & $0.8 \pm 0.4$ & $2.6 \pm 0.5$ \\
$10.75-11.50$ & $0.7 \pm 0.4$ & $2.4 \pm 0.6$ & & $0.5 \pm 0.2$ & \\
\hline
\end{tabular}

Notes. The equation we used is $\operatorname{sBHAR}(\mathrm{sSFR})=\delta(1+z)^{\gamma}$.

to form stars, regardless of stellar mass. From this it follows that $M_{\mathrm{BH}}$ has a superlinear dependence on $M_{*}$ in star-forming and starburst galaxies, and the dependence is linear in quiescent galaxies.

5. sBHAR and sSFR follow very similar decreasing trends in time. We see signs of downsizing in all types of galaxies, a faster accretion (of $M_{\mathrm{BH}}$ and $M_{*}$ ) in starbursts followed by star-forming galaxies, and finally, by quiescent galaxies with mass-doubling timescales of about a Hubble time.

6. The resulting $M_{\mathrm{BH}}-M_{*}$ relation from our data agrees well with independent determinations of the relation that were retrieved from AGN samples and Monte Carlo simulations.

All of these results confirm the coevolution of host galaxy and black hole follows the pattern of downsizing at all redshifts and in different galaxy evolutionary phases. In this picture, the bulk of the black hole and stellar masses is accreted in galaxies during the main-sequence phase through secular processes, where more massive galaxies are more efficient at accreting the black hole. Starburst episodes play a lesser role for both accretions because only a few galaxies are in this phase and these episodes are only weakly able to enhance black hole accretion at high redshifts. The deeper potential well of more massive and possibly more compact galaxies seems to be playing a role in feeding the black hole more efficiently in star-forming and starburst galaxies. In the quiescent life phase of galaxies, the black hole accretion is not as penalized as the star formation. The gas availability reported by Gobat et al. (2018) means that this gas may not go to star formation because of different galactic properties in the different life phases (e.g., disk and bulge dynamics), but to accrete the black hole. Finally, we find additional evidence that suggests that the $M_{\mathrm{BH}}-M_{*}$ may have a lower normalization than the local dynamical relation.

Acknowledgements. The authors want to thank the anonymous referee for his/her comments that clearly improved the consistency and overall quality of this paper. We acknowledge helpful conversations with Patricia Arévalo, Francesca Civano, Francesca M. Fornasini, Mauro Giavalisco, Alister Graham and Claudio Ricci. RC acknowledges financial support from CONICYT Doctorado Nacional $N^{\circ} 21161487$ and CONICYT PIA ACT172033; RC and PC acknowledge support from the CONICYT/FONDECYT program $N^{\circ} 1150216$; GR and CM acknowledge support from an INAF PRIN-SKA 2017 grant 1.05.01.88.04 and from the STARS@UniPD grant; PC acknowledges support from the BIRD 2018 research grant from the Università degli Studi di Padova;
ID is supported by the European Union's Horizon 2020 research and innovation program under the Marie Skłodowska-Curie grant agreement $N^{\circ} 78867$; FS acknowledges partial support from a Leverhulme Trust Research Fellowship.

\section{References}

Aird, J., Coil, A. L., Moustakas, J., et al. 2012, ApJ, 746, 90

Aird, J., Coil, A. L., \& Georgakakis, A. 2017, MNRAS, 465, 3390

Aird, J., Coil, A. L., \& Georgakakis, A. 2018, MNRAS, 474, 1225

Aird, J., Coil, A. L., \& Georgakakis, A. 2019, MNRAS, 484, 4360

Ananna, T. T., Treister, E., Urry, C. M., et al. 2019, ApJ, 871, 240

Arnouts, S., Moscardini, L., Vanzella, E., et al. 2002, MNRAS, 329, 355

Arnouts, S., Le Floc'h, E., Chevallard, J., et al. 2013, A\&A, 558, A67

Ashby, M. L. N., Willner, S. P., Fazio, G. G., et al. 2015, ApJS, 218, 33

Aversa, R., Lapi, A., de Zotti, G., Shankar, F., \& Danese, L. 2015, ApJ, 810, 74 Azadi, M., Aird, J., Coil, A. L., et al. 2015, ApJ, 806, 187

Baronchelli, I., Rodighiero, G., Teplitz, H. I., et al. 2018, ApJ, 857, 64

Barro, G., Faber, S. M., Koo, D. C., et al. 2017, ApJ, 840, 47

Barro, G., Pérez-González, P. G., Cava, A., et al. 2019, ApJS, 243, 22

Bell, E. F., McIntosh, D. H., Katz, N., \& Weinberg, M. D. 2003, ApJ, 585, L117

Bernardi, M., Sheth, R. K., Tundo, E., \& Hyde, J. B. 2007, ApJ, 660, 267

Berta, S., Magnelli, B., Lutz, D., et al. 2010, A\&A, 518, L30

Berta, S., Magnelli, B., Nordon, R., et al. 2011, A\&A, 532, A49

Berta, S., Lutz, D., Santini, P., et al. 2013, A\&A, 551, A100

Bertin, E. 2013, PSFEx: Point Spread Function Extractor

Béthermin, M., Daddi, E., Magdis, G., et al. 2015, A\&A, 573, A113

Bolzonella, M., Miralles, J. M., \& Pelló, R. 2000, A\&A, 363, 476

Bolzonella, M., Kovač, K., Pozzetti, L., et al. 2010, A\&A, 524, A76

Bongiorno, A., Merloni, A., Brusa, M., et al. 2012, MNRAS, 427, 3103

Boyle, B. J., \& Terlevich, R. J. 1998, MNRAS, 293, L49

Brandt, W. N., \& Alexander, D. M. 2015, A\&ARv, 23, 1

Brinchmann, J., Charlot, S., White, S. D. M., et al. 2004, MNRAS, 351, 1151

Brusa, M., Comastri, A., Daddi, E., et al. 2005, A\&A, 432, 69

Bruzual, G., \& Charlot, S. 2003, MNRAS, 344, 1000

Calzetti, D., Armus, L., Bohlin, R. C., et al. 2000, ApJ, 533, 682

Caputi, K. I., Deshmukh, S., Ashby, M. L. N., et al. 2017, ApJ, 849, 45

Chabrier, G. 2003, PASP, 115, 763

Chary, R., \& Elbaz, D. 2001, ApJ, 556, 562

Ciliegi, P., Gruppioni, C., McMahon, R., \& Rowan-Robinson, M. 2001, Ap\&SS, 276,957

Cimatti, A., Daddi, E., \& Renzini, A. 2006, A\&A, 453, L29

Cimatti, A., Cassata, P., Pozzetti, L., et al. 2008, A\&A, 482, 21

Civano, F., Elvis, M., Brusa, M., et al. 2012, ApJS, 201, 30

Civano, F., Marchesi, S., Comastri, A., et al. 2016, ApJ, 819, 62

Corral, A., Georgantopoulos, I., Comastri, A., et al. 2016, A\&A, 592, A109

Cowie, L. L., Songaila, A., Hu, E. M., \& Cohen, J. G. 1996, AJ, 112, 839

da Cunha, E., Charlot, S., \& Elbaz, D. 2008, MNRAS, 388, 1595

da Cunha, E., Eminian, C., Charlot, S., \& Blaizot, J. 2010, MNRAS, 403, 1894

Daddi, E., Dickinson, M., Morrison, G., et al. 2007, ApJ, 670, 156

Delvecchio, I., Gruppioni, C., Pozzi, F., et al. 2014, MNRAS, 439, 2736 
Delvecchio, I., Daddi, E., Shankar, F., et al. 2019, ApJ, 885, L36 Di Matteo, T., Springel, V., \& Hernquist, L. 2005, Nature, 433, 604 Ding, X., Silverman, J., Treu, T., et al. 2020, ApJ, 888, 37 Draine, B. T., \& Li, A. 2007, ApJ, 657, 810

Elbaz, D., Daddi, E., Le Borgne, D., et al. 2007, A\&A, 468, 33

Fang, J. J., Faber, S. M., Koo, D. C., \& Dekel, A. 2013, ApJ, 776, 63

Feldmann, R., Hopkins, P. F., Quataert, E., Faucher-Giguère, C.-A., \& Kereš, D. 2016, MNRAS, 458, L14

Feltre, A., Hatziminaoglou, E., Fritz, J., \& Franceschini, A. 2012, MNRAS, 426 120

Fiore, F., Grazian, A., Santini, P., et al. 2008, ApJ, 672, 94

Fornasini, F. M., Civano, F., Fabbiano, G., et al. 2018, ApJ, 865, 43

Fritz, J., Franceschini, A., \& Hatziminaoglou, E. 2006, MNRAS, 366, 767

Gobat, R., Daddi, E., Magdis, G., et al. 2018, Nat. Astron., 2, 239

Granato, G. L., De Zotti, G., Silva, L., Bressan, A., \& Danese, L. 2004, ApJ, 600,580

Gruppioni, C., Pozzi, F., Andreani, P., et al. 2010, A\&A, 518, L27

Gruppioni, C., Pozzi, F., Rodighiero, G., et al. 2013, MNRAS, 432, 23

Gruppioni, C., Calura, F., Pozzi, F., et al. 2015, MNRAS, 451, 3419

Hopkins, P. F., \& Quataert, E. 2010, MNRAS, 407, 1529

Hopkins, P. F., Hernquist, L., Cox, T. J., \& Kereš, D. 2008, ApJS, 175, 356

Hsieh, B.-C., Wang, W.-H., Hsieh, C.-C., et al. 2012, ApJS, 203, 23

Ilbert, O., Arnouts, S., McCracken, H. J., et al. 2006, A\&A, 457, 841

Ilbert, O., Capak, P., Salvato, M., et al. 2009, ApJ, 690, 1236

Ilbert, O., Salvato, M., Le Floc'h, E., et al. 2010, ApJ, 709, 644

Ilbert, O., McCracken, H. J., Le Fèvre, O., et al. 2013, A\&A, 556, A55

Ilbert, O., Arnouts, S., Le Floc'h, E., et al. 2015, A\&A, 579, A2

Kalberla, P. M. W., Burton, W. B., Hartmann, D., et al. 2005, A\&A, 440, 775

Kennicutt, R. C., Jr 1998, ARA\&A, 36, 189

Koekemoer, A. M., Aussel, H., Calzetti, D., et al. 2007, ApJS, 172, 196

Laigle, C., McCracken, H. J., Ilbert, O., et al. 2016, ApJS, 224, 24

Lapi, A., Shankar, F., Mao, J., et al. 2006, ApJ, 650, 42

Leauthaud, A., Massey, R., Kneib, J.-P., et al. 2007, ApJS, 172, 219

Le Floc'h, E., Aussel, H., Ilbert, O., et al. 2009, ApJ, 703, 222

Lehmer, B. D., Basu-Zych, A. R., Mineo, S., et al. 2016, ApJ, 825, 7

Lusso, E., Comastri, A., Simmons, B. D., et al. 2012, MNRAS, 425, 623

Lutz, D., Poglitsch, A., Altieri, B., et al. 2011, A\&A, 532, A90

Madau, P., \& Dickinson, M. 2014, ARA\&A, 52, 415

Marchesi, S., Civano, F., Elvis, M., et al. 2016, ApJ, 817, 34

Marconi, A., Risaliti, G., Gilli, R., et al. 2004, MNRAS, 351, 169

Markwardt, C. B. 2009, in Astronomical Data Analysis Software and Systems XVIII, eds. D. A. Bohlender, D. Durand, \& P. Dowler, ASP Conf. Ser., 411, 251

McCracken, H. J., Capak, P., Salvato, M., et al. 2010, ApJ, 708, 202

McCracken, H. J., Milvang-Jensen, B., Dunlop, J., et al. 2012, A\&A, 544, A156

Merloni, A., \& Heinz, S. 2008, MNRAS, 388, 1011

Mineo, S., Gilfanov, M., \& Sunyaev, R. 2012, MNRAS, 426, 1870

Miyaji, T., \& Griffiths, R. E. C-COSMOS Team 2008, AAS/High Energy Astrophysics Division \# 10, AAS/High Energy Astrophysics Division, 4.01 4.01
Mullaney, J. R., Daddi, E., Béthermin, M., et al. 2012a, ApJ, 753, L30 Mullaney, J. R., Pannella, M., Daddi, E., et al. 2012b, MNRAS, 419, 95 Noeske, K. G., Weiner, B. J., Faber, S. M., et al. 2007, ApJ, 660, L43 Nordon, R., Lutz, D., Saintonge, A., et al. 2013, ApJ, 762, 125

Oliver, S. J., Bock, J., Altieri, B., et al. 2012, MNRAS, 424, 1614 Paggi, A., Fabbiano, G., Civano, F., et al. 2016, ApJ, 823, 112 Polletta, M., Tajer, M., Maraschi, L., et al. 2007, ApJ, 663, 81 Reines, A. E., \& Volonteri, M. 2015, ApJ, 813, 82

Ricci, C., Ueda, Y., Koss, M. J., et al. 2015, ApJ, 815, L13

Rieke, G. H., Alonso-Herrero, A., Weiner, B. J., et al. 2009, ApJ, 692, 556 Riguccini, L. A., Treister, E., Menéndez-Delmestre, K., et al. 2019, ApJ, 157, 233

Rodighiero, G., Daddi, E., Baronchelli, I., et al. 2011, ApJ, 739, L40 Rodighiero, G., Renzini, A., Daddi, E., et al. 2014, MNRAS, 443, 19 Rodighiero, G., Brusa, M., Daddi, E., et al. 2015, ApJ, 800, L10 Rodighiero, G., Enia, A., Delvecchio, I., et al. 2019, ApJ, 877, L38 Rosario, D. J., Santini, P., Lutz, D., et al. 2012, A\&A, 545, A45 Sahu, N., Graham, A. W., \& Davis, B. L. 2019, ApJ, 876, 155 Salvato, M., Ilbert, O., Hasinger, G., et al. 2011, ApJ, 742, 61 Sanders, D. B., Salvato, M., Aussel, H., et al. 2007, ApJS, 172, 86 Santini, P., Ferguson, H. C., Fontana, A., et al. 2015, ApJ, 801, 97 Sargent, M. T., Béthermin, M., Daddi, E., \& Elbaz, D. 2012, ApJ, 747, L31 Savorgnan, G. A. D., \& Graham, A. W. 2016, ApJS, 222, 10 Schreiber, C., Pannella, M., Elbaz, D., et al. 2015, A\&A, 575, A74 Scoville, N., Aussel, H., Brusa, M., et al. 2007, ApJS, 172, 1 Shankar, F., Salucci, P., Granato, G. L., De Zotti, G., \& Danese, L. 2004, MNRAS, 354, 1020

Shankar, F., Weinberg, D. H., \& Miralda-Escudé, J. 2009, ApJ, 690, 20 Shankar, F., Bernardi, M., Sheth, R. K., et al. 2016, MNRAS, 460, 3119 Shankar, F., Bernardi, M., \& Sheth, R. K. 2017, MNRAS, 466, 4029 Shankar, F., Bernardi, M., Richardson, K., et al. 2019, MNRAS, 485, 1278 Shankar, F., Weinberg, D. H., Marsden, C., et al. 2020, MNRAS, 493, 1500 Shao, L., Lutz, D., Nordon, R., et al. 2010, A\&A, 518, L26 She, R., Ho, L. C., \& Feng, H. 2017, ApJ, 842, 131

Silverman, J. D., Lamareille, F., Maier, C., et al. 2009, ApJ, 696, 396 Somerville, R. S., \& Davé, R. 2015, ARA\&A, 53, 51 Suh, H., Civano, F., Hasinger, G., et al. 2019, ApJ, 872, 168 Suh, H., Civano, F., Trakhtenbrot, B., et al. 2020, ApJ, 889, 32 Sutherland, W., \& Saunders, W. 1992, MNRAS, 259, 413 Szalay, A. S., Connolly, A. J., \& Szokoly, G. P. 1999, AJ, 117, 68

Taniguchi, Y., Scoville, N., Murayama, T., et al. 2007, ApJS, 172, 9 Taniguchi, Y., Kajisawa, M., Kobayashi, M. A. R., et al. 2015, PASJ, 67, 104 Tasca, L. A. M., Le Fèvre, O., Hathi, N. P., et al. 2015, A\&A, 581, A54 Tomczak, A. R., Quadri, R. F., Tran, K.-V. H., et al. 2016, ApJ, 817, 118 Ueda, Y., Akiyama, M., Hasinger, G., Miyaji, T., \& Watson, M. G. 2014, ApJ, 786, 104

Vito, F., Gilli, R., Vignali, C., et al. 2014, MNRAS, 445, 3557

Yang, G., Chen, C. T. J., Vito, F., et al. 2017, ApJ, 842, 72

Yang, G., Brandt, W. N., Vito, F., et al. 2018, MNRAS, 475, 1887

Zamojski, M. A., Schiminovich, D., Rich, R. M., et al. 2007, ApJS, 172, 468 


\section{Appendix A: Sample properties}

We report the tables with additional properties of our sample.

Table A.1 lists star-forming galaxies, Table A.2 quiescent galaxies, and Table A.3 starbursts.

Table A.1. Properties of the star-forming galaxy sample.

\begin{tabular}{lccccccc}
\hline \hline $\begin{array}{l}\text { Mass range } \\
\log _{10}\left(M_{*} / M_{\odot}\right)\end{array}$ & $\begin{array}{c}M_{*} \\
\log _{10}\left(M_{*} / M_{\odot}\right)\end{array}$ & $z$ & $N_{\text {stacked }}$ & $N_{\text {detected }}$ & $\begin{array}{c}L_{2-10 \mathrm{keV}} \\
10^{42} \mathrm{erg} \mathrm{s}^{-1}\end{array}$ & $\begin{array}{c}\mathrm{SFR}_{\mathrm{IR}} \\
M_{\odot} \mathrm{yr}^{-1}\end{array}$ & $\begin{array}{c}\mathrm{SFR}_{\mathrm{UV}} \\
M_{\odot} \mathrm{yr}^{-1}\end{array}$ \\
\hline $0.10<z<0.65$ & & & & & & & \\
\hline $9.5-10.0$ & 9.72 & 0.46 & 4244 & 19 & $0.023_{-0.007}^{+0.007}$ & $2.22_{-0.07}^{+0.08}$ & $0.500_{-0.011}^{+0.012}$ \\
$10.0-10.5$ & 10.21 & 0.45 & 2514 & 75 & $0.142_{-0.009}^{+0.009}$ & $5.68_{-0.17}^{+2.67}$ & $0.479_{-0.018}^{+0.013}$ \\
$10.5-11.0$ & 10.70 & 0.45 & 1214 & 117 & $0.639_{-0.015}^{+0.015}$ & $14.2_{-0.7}^{+0.7}$ & $0.56_{-0.02}^{+0.03}$ \\
$11.0-12.0$ & 11.14 & 0.43 & 239 & 38 & $1.550_{-0.036}^{+0.038}$ & $15.7_{-2.4}^{+2.2}$ & $0.76_{-0.11}^{+0.07}$ \\
\hline $0.65<z<1.30$ & & & & & & & \\
\hline $9.5-10.0$ & 9.72 & 0.97 & 15431 & 56 & $0.15_{-0.02}^{+0.02}$ & $4.70_{-0.13}^{+0.14}$ & $1.051_{-0.011}^{+0.009}$ \\
$10.0-10.5$ & 10.22 & 0.97 & 7957 & 175 & $0.66_{-0.03}^{+0.03}$ & $14.9_{-0.3}^{+0.3}$ & $0.854_{-0.015}^{+0.018}$ \\
$10.5-11.0$ & 10.69 & 0.95 & 3888 & 370 & $2.67_{-0.05}^{+0.05}$ & $26.8_{-0.5}^{+0.5}$ & $0.856_{-0.016}^{+0.023}$ \\
$11.0-12.0$ & 11.15 & 0.95 & 761 & 142 & $5.13_{-0.12}^{+0.12}$ & $37.1_{-2.1}^{+2.7}$ & $1.29_{-0.13}^{+0.08}$ \\
\hline $1.30<z<2.25$ & & & & & & & \\
\hline $9.5-10.0$ & 9.80 & 1.64 & 13451 & 47 & $0.35_{-0.08}^{+0.09}$ & $10.4_{-0.6}^{+0.6}$ & $2.17_{-0.02}^{+0.02}$ \\
$10.0-10.5$ & 10.22 & 1.73 & 11334 & 177 & $1.47_{-0.10}^{+0.09}$ & $30.2_{-0.7}^{+0.7}$ & $1.62_{-0.03}^{+0.03}$ \\
$10.5-11.0$ & 10.69 & 1.74 & 5273 & 368 & $5.97_{-0.16}^{+0.17}$ & $57.0_{-1.5}^{+1.5}$ & $1.18_{-0.02}^{+0.02}$ \\
$11.0-12.0$ & 11.12 & 1.76 & 954 & 172 & $18.21_{-0.45}^{+0.43}$ & $97.0_{-4.7}^{+4.6}$ & $1.46_{-0.06}^{+0.04}$ \\
\hline $2.25<z<3.50$ & & & & & & & \\
\hline $9.5-10.0$ & 9.92 & 2.69 & 1264 & 3 & $0.8_{-0.9}^{+0.8}$ & $14.9_{-6.7}^{+8.6}$ & $4.72_{-0.17}^{+0.15}$ \\
$10.0-10.5$ & 10.22 & 2.77 & 7159 & 98 & $2.9_{-0.4}^{+0.4}$ & $68.2_{-4.1}^{+4.4}$ & $4.49_{-0.08}^{+0.07}$ \\
$10.5-11.0$ & 10.68 & 2.73 & 2726 & 166 & $14.9_{-0.6}^{+0.6}$ & $139.4_{-9.1}^{+9.1}$ & $2.78_{-0.05}^{+0.006}$ \\
$11.0-12.0$ & 11.13 & 2.69 & 466 & 70 & $35.5_{-1.5}^{+1.6}$ & $243.4_{-14.8}^{+16.3}$ & $2.44_{-0.14}^{+0.12}$ \\
\hline
\end{tabular}

Notes. For each mass and redshift bin we show the median mass and redshift, and the number of stacked and detected galaxies in the 2-7 keV band. We also show the X-ray luminosity, the SFR estimated from the FIR, and the obscured SFR from the UV. Quantities are medians, and confidence ranges are $1 \sigma$. 
R. Carraro et al.: Coevolution of black hole accretion and star formation in galaxies up to $z=3.5$

Table A.2. Same as Table A.1 for the quiescent galaxy sample.

\begin{tabular}{lccccccc}
\hline \hline $\begin{array}{l}\text { Mass range } \\
\log _{10}\left(M_{*} / M_{\odot}\right)\end{array}$ & $\begin{array}{c}M_{*} \\
\log _{10}\left(M_{*} / M_{\odot}\right)\end{array}$ & $z$ & $N_{\text {stacked }}$ & $N_{\text {detected }}$ & $\begin{array}{c}L_{2-10 \mathrm{keV}} \\
10^{42} \mathrm{erg} \mathrm{s}^{-1}\end{array}$ & $\begin{array}{c}\mathrm{SFR}_{\mathrm{IR}} \\
M_{\odot} \mathrm{yr}^{-1}\end{array}$ & $\begin{array}{c}\mathrm{SFR}_{\mathrm{UV}} \\
M_{\odot} \mathrm{yr}^{-1}\end{array}$ \\
\hline $0.10<z<0.65$ & & & & & & & \\
\hline $10.0-10.5$ & 10.27 & 0.47 & 867 & 12 & $0.060_{-0.018}^{+0.017}$ & $0.40_{-0.09}^{+0.07}$ & $0.017_{-0.002}^{+0.002}$ \\
$10.5-11.0$ & 10.74 & 0.47 & 830 & 19 & $0.061_{-0.015}^{+0.015}$ & $0.51_{-0.10}^{+0.12}$ & $0.026_{-0.004}^{+0.004}$ \\
$11.0-12.0$ & 11.16 & 0.50 & 271 & 14 & $0.177_{-0.035}^{+0.035}$ & $0.96_{-0.22}^{+0.21}$ & $0.074_{-0.017}^{+0.010}$ \\
\hline $0.65<z<1.30$ & & & & & & & \\
\hline $10.0-10.5$ & 10.30 & 0.93 & 2594 & 19 & $0.18_{-0.05}^{+0.04}$ & $1.3_{-0.4}^{+0.3}$ & $0.083_{-0.002}^{+0.002}$ \\
$10.5-11.0$ & 10.75 & 0.94 & 3354 & 72 & $0.41_{-0.04}^{+0.04}$ & $2.2_{-0.2}^{+0.3}$ & $0.130_{-0.003}^{+0.003}$ \\
$11.0-12.0$ & 11.15 & 0.93 & 1107 & 25 & $0.47_{-0.08}^{+0.08}$ & $2.8_{-0.4}^{+0.5}$ & $0.172_{-0.010}^{+0.009}$ \\
\hline $1.30<z<2.25$ & & & & & & & \\
\hline $10.0-10.5$ & 10.25 & 1.58 & 769 & 9 & $0.6_{-0.3}^{+0.3}$ & - & $0.279_{-0.011}^{+0.013}$ \\
$10.5-11.0$ & 10.75 & 1.57 & 1534 & 25 & $1.2_{-0.2}^{+0.2}$ & $7.4_{-0.8}^{+0.8}$ & $0.307_{-0.008}^{+0.008}$ \\
$11.0-12.0$ & 11.14 & 1.59 & 532 & 8 & $1.8_{-0.4}^{+0.4}$ & $6.0_{-3.0}^{+2.1}$ & $0.400_{-0.012}^{+0.021}$ \\
\hline $2.25<z<3.50$ & & & & & & & \\
\hline $10.0-10.5$ & 10.32 & 2.56 & 105 & 3 & $8.4_{-2.6}^{+2.5}$ & - & $1.25_{-0.06}^{+0.22}$ \\
$10.5-11.0$ & 10.75 & 2.52 & 272 & 7 & $2.6_{-1.5}^{+1.5}$ & - & $1.15_{-0.07}^{+0.06}$ \\
$11.0-12.0$ & 11.12 & 2.48 & 66 & 6 & $22.2_{-3.6}^{+3.6}$ & - & $1.71_{-0.15}^{+0.18}$ \\
\hline
\end{tabular}

Table A.3. Same as Table A.1 for the starburst galaxy sample. We only use $\mathrm{SFR}_{\mathrm{IR}}$ for this sample.

\begin{tabular}{lcccccc}
\hline \hline $\begin{array}{l}\text { Mass range } \\
\log _{10}\left(M_{*} / M_{\odot}\right)\end{array}$ & $\begin{array}{c}M_{*} \\
\log _{10}\left(M_{*} / M_{\odot}\right)\end{array}$ & $z$ & $N_{\text {stacked }}$ & $N_{\text {detected }}$ & $\begin{array}{c}L_{2-10 \mathrm{keV}} \\
10^{42} \mathrm{erg} \mathrm{s}^{-1}\end{array}$ & $\begin{array}{c}\mathrm{SFR}_{\mathrm{IR}} \\
M_{\odot} \mathrm{yr}^{-1}\end{array}$ \\
\hline $0.10<z<0.65$ & & & & & & \\
\hline $9.50-10.25$ & 9.87 & 0.53 & 58 & 4 & $0.36_{-0.09}^{+0.09}$ & $22_{-2}^{+4}$ \\
$10.25-10.75$ & 10.52 & 0.53 & 6 & 5 & $4.68_{-0.41}^{+0.40}$ & $74_{-9}^{+20}$ \\
$10.75-11.50$ & 10.96 & 0.52 & 2 & 3 & $6.64_{-0.68}^{+0.67}$ & $143_{-1}^{+5}$ \\
\hline $0.65<z<1.30$ & & & & & & \\
\hline $9.50-10.25$ & 9.90 & 0.95 & 162 & 7 & $0.8_{-0.2}^{+0.2}$ & $66_{-3}^{+3}$ \\
$10.25-10.75$ & 10.42 & 1.04 & 161 & 10 & $2.5_{-0.3}^{+0.3}$ & $131_{-2}^{+5}$ \\
$10.75-11.50$ & 10.91 & 1.08 & 43 & 5 & $6.2_{-0.6}^{+0.6}$ & $280_{-16}^{+15}$ \\
\hline $1.30<z<2.25$ & & & & & & \\
\hline $9.50-10.25$ & 10.01 & 1.76 & 57 & 1 & & $222_{-18}^{+15}$ \\
$10.25-10.75$ & 10.50 & 1.74 & 145 & 24 & $15.1_{-1.2}^{+1.2}$ & $331_{-9}^{+16}$ \\
$10.75-11.50$ & 10.89 & 1.79 & 78 & 23 & $40.8_{-2.0}^{+2.0}$ & $536_{-23}^{+34}$ \\
\hline $2.25<z<3.50$ & & & & & & \\
\hline $9.50-10.25$ & 10.07 & 2.48 & 5 & 0 & & - \\
$10.25-10.75$ & 10.53 & 2.59 & 64 & 2 & $8.3_{-4.1}^{+4.5}$ & $739_{-26}^{+27}$ \\
$10.75-11.50$ & 10.90 & 2.68 & 46 & 7 & $20.1_{-5.3}^{+5.5}$ & $1229_{-139}^{+148}$ \\
\hline
\end{tabular}

